\title{
The non-LTE line formation of neutral carbon in late-type stars
}

\author{
D. Fabbian ${ }^{1}$, M. Asplund ${ }^{1}$, M. Carlsson ${ }^{2,3}$, and D. Kiselman ${ }^{4}$ \\ ${ }^{1}$ Research School of Astronomy \& Astrophysics, The Australian National University, Mount Stromlo Observatory, Cotter Road, \\ Weston ACT 2611, Australia \\ e-mail: damian@mso.anu.edu.au \\ 2 Institute of Theoretical Astrophysics, University of Oslo, PO Box 1029, Blindern, 0315 Oslo, Norway \\ 3 Center of Mathematics for Applications, University of Oslo, PO Box 1053 Blindern, 0316 Oslo, Norway \\ 4 The Institute for Solar Physics of the Royal Swedish Academy of Sciences, AlbaNova University Centre, 10691 Stockholm, \\ Sweden
}

Received 6 June 2006 / Accepted 27 July 2006

ABSTRACT

\begin{abstract}
Aims. We investigate the non-Local Thermodynamic Equilibrium (non-LTE) line formation of neutral carbon in late-type stars in order to remove some of the potential systematic errors in stellar abundance analyses employing C I features.

Methods. The statistical equilibrium code MULTI was used on a grid of plane-parallel 1D MARCS atmospheric models.

Results. Within the parameter space explored, the high-excitation C I lines studied are stronger in non-LTE due to the combined effect of line source function drop and increased line opacity due to overpopulation of the lower level for the transitions considered; the relative importance of the two effects depends on the particular combination of $T_{\text {eff }}, \log g,[\mathrm{Fe} / \mathrm{H}]$ and $[\mathrm{C} / \mathrm{Fe}]$ and on the analysed C I line. As a consequence, the non-LTE abundance corrections are negative and can be substantially so, for example $\sim-0.4$ dex in halo turn-off stars at $[\mathrm{Fe} / \mathrm{H}] \sim-3$. The magnitude of the non-LTE corrections is rather insensitive to whether inelastic $\mathrm{H}$ collisions are included or not.

Conclusions. Our results have implications on studies of nucleosynthetic processes and on Galactic chemical evolution models. When applying our calculated corrections to recent observational data, the upturn in $[\mathrm{C} / \mathrm{O}]$ at low metallicity might still be present (thus apparently still necessitating contributions from massive Pop. III stars for the carbon production), but at a lower level and possibly with a rather shallow trend of $\sim-0.2 \mathrm{dex} / \mathrm{dex}$ below $[\mathrm{O} / \mathrm{H}] \sim-1$.
\end{abstract}

Key words. line: formation - stars: abundances - stars: late-type - Galaxy: abundances - Galaxy: evolution

\section{Introduction}

Carbon is synthesised through the $3 \alpha$ nuclear reaction in stellar interiors (Burbidge et al. 1957; Arnett 1996; Pagel 1997). It is one of the most abundant elements in the Universe, together with hydrogen, helium, oxygen and neon. It can be found in a variety of compounds, so that it forms the basis of organic chemistry and of life (at least as we know it). Carbon is also important for dust formation processes in the interstellar medium (e.g. Dopita \& Sutherland 2003). Since it has a quite high ionization potential $(11.26 \mathrm{eV})$, it remains mostly neutral in the photosphere of solartype stars. Stellar spectroscopists thus rely on high-excitation permitted C I features and on the forbidden [C I] line at $872.7 \mathrm{~nm}$, as well as on various molecular species (mainly $\mathrm{CH}$ and $\mathrm{C}_{2}$ ), to derive solar and stellar C abundances (e.g. Asplund et al. 2005a).

A number of studies have investigated the carbon content of disk stars in the Galaxy (e.g. Andersson \& Edvarsson 1994; Gustafsson et al. 1999), arguing that $[\mathrm{C} / \mathrm{Fe}]^{1}$ is slowly decreasing with time (i.e. with increasing metallicity) and probably mostly contributed by massive stars (MSs). In particular, Gustafsson et al. rule out a main origin of carbon in low-mass stars while they argue that the contribution of intermediate-mass stars to the production of carbon in the Galaxy is unclear. Takeda \& Honda (2005) have recently studied the CNO abundances of 160 disk F,

1 The usual spectroscopic notation $[\mathrm{A} / \mathrm{X}]=\log \left(N_{\mathrm{A}} / N_{\mathrm{X}}\right)_{*}-$ $\log \left(N_{\mathrm{A}} / N_{\mathrm{X}}\right)_{\odot}$ is used throughout.
$\mathrm{G}$, and $\mathrm{K}$ dwarfs and subgiants with $-1<[\mathrm{Fe} / \mathrm{H}]<+0.4$ to look for any differences between the abundances of stars with and without planets. In particular, when taking their calculated non-LTE corrections into account, they also found that $[\mathrm{C} / \mathrm{Fe}]$ progressively decreases with increasing metallicity for these galactic disk stars, by around $\sim 0.2$ dex per dex. A similar slope has been found for [C/Fe] by Reddy et al. $(2003,2006)$ in their analysis of a number of elements in a large sample of $F$ and $\mathrm{G}$ dwarfs in the thin- and thick-disk. In contrast with the above authors, Bensby \& Feltzing (2006) find essentially no trend of $[\mathrm{C} / \mathrm{Fe}]$ versus $[\mathrm{Fe} / \mathrm{H}]$ for metal-rich thick- and thin-disk stars and conclude that the carbon enrichment at such metallicities is mainly due to low- and intermediate-mass stars (LIMSs), with massive stars still playing the role of main contributors at lower metallicities in the thick disk/halo.

The halo stellar population is less well studied. It is still currently a matter of debate whether for halo stars carbon is produced primarily in LIMSs (e.g. Chiappini et al. 2003), or in massive stars (e.g. Akerman et al. 2004). It could well be that contributions from both of these sources operating on different timescales are important. Akerman et al. were the first to derive a previously unrecognized $[\mathrm{C} / \mathrm{O}]$ upturn at low metallicities. However, they warned about possible non-LTE effects at low metallicity for the high-excitation C I lines they employed and cautioned that their results might be prone to uncertainties due to their assumption that C I-based abundances would be 
affected by the same non-LTE corrections as for O I (which they estimated for their sample by interpolating between the values given in Nissen et al. 2002) and thus that the [C/O] ratio would be unchanged with respect to LTE. They mentioned the possibility that non-LTE effects for the carbon and oxygen highexcitation lines might differ by an amount increasing with decreasing metallicity. At that stage, they were not able to exclude that the $[\mathrm{C} / \mathrm{O}]$ values were being overestimated by $\sim 0.2$ dex under the assumption of LTE. The idea that the upturn could thus be removed is in fact presented in some recent work (e.g. Takeda \& Honda 2005; Fabbian et al. 2005; Asplund 2005; Bensby \& Feltzing 2006). Spite et al. (2005) study a sample of extremely metal-poor giants to investigate the abundances of $\mathrm{C}, \mathrm{N}, \mathrm{O}$ and Li. They find that in their "unmixed" stars (which should reflect the abundances in the early Galaxy) the $[\mathrm{C} / \mathrm{Fe}]$ ratio is remarkably constant (at a level of $\sim+0.2-0.3$ dex) over the entire range $-4.0<[\mathrm{Fe} / \mathrm{H}]<-2.5$ and that the $[\mathrm{C} / \mathrm{O}]$ ratio is close to solar at the lowest metallicities. According to the studies of Akerman et al. (2004) and Spite et al. (2005), the upturn in the $[\mathrm{C} / \mathrm{O}]$ abundances of halo stars present at low metallicity could signal substantial early nucleosynthetic production of carbon in massive Pop. III stars. However it is worth noting that the inclusion of non-LTE/3D effects plays a substantial role in testing such hypothesis. For example, $3 \mathrm{D}$ effects affecting the $\mathrm{C}$ and $\mathrm{O}$ abundance indicators used in the study by Spite et al. (2005) might reach $\sim-0.6$ dex or more for $\mathrm{CH}$, while they likely remain around -0.2 dex for the [OI] forbidden oxygen lines (Asplund 2005; see also Collet et al. 2006). This would then make their resulting $[\mathrm{C} / \mathrm{O}]$ trend more or less flat at a level of $\sim-0.6 \mathrm{dex}$ down to very low $[\mathrm{O} / \mathrm{H}]$ ratios, implying that resorting to high yields from massive zero-metallicity stars might be premature.

On the theoretical side, low-metallicity fast-rotating models of massive stars (Meynet et al. 2006; see also Chiappini et al. 2006) predict important CNO yields through large mass loss during the red supergiant phase via stellar winds caused by efficient mixing and dredge-up enriching the stellar surface in heavy elements. These yields, especially if used in conjuction with a top-heavy IMF, would thus give large carbon abundances at low metallicity without introducing Pop. III stars. Carigi et al. (2005) have constructed various Galactic chemical evolution models with different $\mathrm{C}, \mathrm{N}$ and $\mathrm{O}$ yields. The only models that are able to fit the carbon gradient are those including $\mathrm{C}$ yields that increase with metallicity due to stellar winds in massive stars and decrease with metallicity due to stellar winds in LIMSs. They argue that the fraction of carbon in the interstellar medium due to MSs and LIMSs would thus be dependent on time (with MSs dominating the $\mathrm{C}$ enrichment at early times, while around $12+\log (\mathrm{O} / \mathrm{H}) \sim 8$ the LIMSs formed in the halo should be at the end of their evolution and they would start contributing with $\mathrm{C}$ ejecta in a comparable amount to that of MSs, see also Bensby \& Feltzing 2006) and on galactocentric distance (LIMSs giving a larger contribution at large $r_{\mathrm{GC}}$ ), with similar contribution from both sources to the present carbon abundance in the interstellar medium of the solar vicinity. However, no attempt was made in that work to homogenize the sample of available observational data. Moreover, their "best" models are still not able to properly fit the $N$ abundance values for metal rich stars and the $\mathrm{C} / \mathrm{O}$ values at intermediate or low metallicity obtained from stellar observations. Gavilán et al. (2005) have recently suggested that a Galactic Chemical Evolution (GCE) model including yields from Woosley \& Weaver (1995) for massive stars needs to be integrated with yields from LIMSs to properly reproduce the wealth of observational data on the $[\mathrm{C} / \mathrm{Fe}]$ trend down to $[\mathrm{Fe} / \mathrm{H}] \sim-2.4$, suggesting that a downward revision of mass loss by stellar winds from massive stars is necessary and adding to the increasing evidence that LIMSs are significant contributors to the Inter Stellar Medium (ISM) carbon enrichment. Interestingly, Ballero et al. (2006) test several sets of yields for very massive zero-metallicity stars, using the two-infall chemical evolution model by Chiappini et al. (1997). They argue that the contribution of massive Population III stars to the Galactic evolution of carbon, nitrogen and iron would be negligible even at the lowest metallicities currently probed and that the existence of such objects could in any case not be proven or excluded on the basis of available data on known metal-poor halo stars, which likely formed as second-generation objects after the explosion of primordial supernovae.

Absorption systems give the opportunity to measure abundances in protogalactic structures at high redshift. For example, Becker et al. (2006) recently detected an overabundance of OI absorption features in spectra of background high-redshift quasars (QSOs) towards the line-of-sight of Lyman- $\alpha$ forest clouds, deriving a mean $\langle[\mathrm{C} / \mathrm{O}]\rangle=-0.31 \pm 0.09$. Moreover, Pettini (private communication) and Erni et al. (2006) also find $[\mathrm{C} / \mathrm{O}] \sim-0.3$ in metal-poor Damped Lyman- $\alpha$ systems (DLAs) with $[\mathrm{O} / \mathrm{H}] \sim-2.5$, in reasonable agreement with the values for Galactic stars (Akerman et al. 2004). These results are obviously precious in complementing Galactic studies at low $[\mathrm{Fe} / \mathrm{H}]$. In particular, Erni et al. argue that enrichment from a population of massive (10-50 $\left.M_{\odot}\right)$ Pop. III stars exploding as corecollapse supernovae (SNeII) and possibly as hyper-energetic $\left(E>10^{51} \mathrm{erg}\right.$ ) so-called hypernovae (HNe) is the most likely scenario, in contrast to models using non-zero metallicity progenitors or other explosion mechanisms, such as super-massive (140-260 $M_{\odot}$ ) pair-instability supernovae (PISNe) or Type Ia supernovae (SNeIa). Levshakov et al. (2006) derive a very low abundance of ${ }^{13} \mathrm{C}$ towards a quasar at $z=1.15$. This does not support the enrichment of gas by fast-rotating, very low- $Z$ massive stars (e.g. Meynet et al. 2006) and puts a tight bound to the possible amount of contributions from intermediate-mass AGB stars to the $\mathrm{C}$ nucleosynthesis in matter sampled by the line of sight to this system.

The still open problem of the origin of carbon in the early Universe constitutes the main motivation for the present nonLTE study. The quality of stellar spectroscopic observations possible at present, require us to make corresponding progress in models of stellar atmospheres and line formation processes in order to remove systematic errors. The impact on Galactic chemical evolution studies of such non-LTE calculations promises to be rewarding.

\section{C I non-LTE line formation}

\subsection{Non-LTE preamble}

Spectral lines are powerful diagnostic tools that provide us with the capability of "remotely sensing" the physical conditions of the matter composing the particular atmosphere in which they form, via the analysis of the radiation we receive from it. However, the problem of how these lines are actually formed when energy is transferred through the atmosphere by radiation, is extremely complex (Mihalas \& Mihalas 2000; Rutten 2003). The abundances of the chemical elements present in the atmospheres of stars are therefore usually derived relying on the simplifying assumption of Local Thermodynamic Equilibrium (LTE) to derive the atomic level occupation numbers (Gray 1992). The visible electromagnetic radiation we observe from stars comes from their photosphere, where radiation-matter 
interaction can play an important role. Radiation is in fact absorbed and emitted by atoms and converted to different wavelengths via the interaction with the matter composing the atmosphere; vice versa the atomic level populations are influenced by radiation. The LTE treatment assumes that this complex process is governed by a single temperature parameter.

However, radiative rates often dominate over collisional ones in the atmospheric layers where the lines are formed and thus the LTE approximation can give misleading results. When LTE is not assumed a priori, the Saha-Boltzmann equation can no longer be used to determine the level populations from local properties. A large number of radiative and collisional processes needs to be treated simultaneously to account for the coupling between the populations of different atomic levels for the particular chemical element of interest. The major non-LTE effects in late-type stars have recently been reviewed by Asplund (2005). The main mechanisms are:

- UV overionization: neutral minority stage elements (like e.g. $\mathrm{Fe} I$ in the Sun) can be affected by overionization triggered by ultraviolet radiation (bound-free pumping). This is caused by a $J_{v}-B_{v}$ radiation excess at short wavelengths (where $J_{v}$ is the angle-averaged mean intensity and $B_{v}$ is the Planck function), with the radiation temperature exceeding the local electron temperature;

- Resonance line scattering and photon losses: scattering from deep layers and photon losses can make the line source function drop below the local Planck function, resulting in nonLTE line strengthening;

- Infrared overrecombination: occurs when $J_{v}$ drops below $B_{v}$ and causes recombination to high-energy-lying states;

- Bound-bound pumping: ultraviolet/optical radiation can cause pumping of bound-bound transitions, increasing the populations of upper levels; it can moreover cause overionisation (like in B I or Li I), since other levels than the one involved in the transition can be directly influenced; and

- Photon suction: caused by a recombination downflow from the first ionization stage, being for example the cause of the observed $12 \mu \mathrm{m}$ emission $\mathrm{Mg}$ I features in the Sun.

\subsection{Model atom}

Given the complex interaction of the different physical mechanisms in the line formation process, the accuracy of the atomic data needed as input is crucial to the final reliability of non-LTE calculations.

Our carbon model atom contains 217 energy levels (207 for C I, 9 for $\mathrm{C} \mathrm{II} \mathrm{and} \mathrm{one} \mathrm{for} \mathrm{C} \mathrm{III)} \mathrm{and} \mathrm{is} \mathrm{constructed} \mathrm{taking} \mathrm{into}$ detailed account a total of 650 radiative transitions (453 boundbound and 197 bound-free). The neutral carbon levels are included up to an excitation potential of $11.13 \mathrm{eV}$ (i.e. $\sim 0.13 \mathrm{eV}$ below the continuum, complete to a principal quantum number of $n=9$ ), the singly ionized levels up to $28.87 \mathrm{eV}$, while the $\mathrm{C}$ III level is at $35.64 \mathrm{eV}$. All radiative transition probabilities ( $f$-values and photo-ionization cross-sections) are taken from the Opacity Project database TOPbase (Cunto et al. 1993; see also, specifically for carbon, Luo \& Pradhan 1989; and Hibbert et al. 1993). The necessary data for central wavelengths, radiative broadening and excitation potential for the atomic energy levels were taken from the NIST ${ }^{2}$ (Martin et al. 1995; Wiese et al. 1996) database.

\footnotetext{
${ }^{2}$ http://physics.nist.gov/PhysRefData/
}

Our model atom also includes collisional excitations and ionizations by electrons and neutral hydrogen atoms. The crosssections for forbidden transitions by electron collisions are derived with van Regemorter's (1962) formula (adopting an oscillator strength $f=0.01$ ), while the impact approximation is employed for radiatively allowed transitions. One of the largest sources of uncertainty in current non-LTE studies is due to the unavailability of theoretical and laboratory collisional crosssections for inelastic collisions with HI. Hydrogen collisions in general act as a thermalizing source, bringing the balance of level populations closer to LTE. Especially in metal-poor stars, where neutral hydrogen atoms outnumber free electrons, such collisions should be of importance (e.g. Asplund 2005). We treated these collisions through the use of Drawin's formula (1968), as generalized by Steenbock \& Holweger (1984) to the case of collisions between different particles.

The Grotrian term diagram for our model atom is shown in Fig. 1. When using this atomic model, we did not include fine structure splitting for the levels of interest here, which means that the level populations were computed with compound lines. We then expect each of these specific "grouped" lines to be stronger (and thus formed in higher atmospheric layers where the non-LTE effects are likely to be somewhat greater) than the corresponding observed splitted lines for the same term transitions. To be able to compare our results with observations, we have used a second model atom that includes fine splitting, redistributing the converged population densities according to statistical weight. Table 1 gives the relevant details for the lines included in this smaller atomic model (see also Fig. 1). Since one could be concerned that non-LTE effects may be overestimated using this approach ${ }^{3}$ we have carried out tests which indicate that only relatively small differences are experienced in terms of abundance corrections when adopting the more consistent approach (the results remaining within \pm 0.05 dex of our standard results at all metallicities, the difference becoming even more negligible towards $[\mathrm{Fe} / \mathrm{H}] \sim-3$ ). Since this marginal difference can be either positive and negative at the different metallicities, it is unlikely to introduce any systematic bias when using our computed non-LTE abundance corrections. In these tests, the radiative and collisional cross-sections in the splitted level case were reduced accordingly to the increased number of levels with respect to the unsplitted case, namely redistributing according to statistical weight, and a smaller 33-level atomic model was used to speed up the calculations, since we found that only small differences are obtained with respect to runs using the full 217-level atom (the results remaining within \pm 0.05 dex at all metallicities). This is indeed a very interesting result and one which we plan to exploit in a future work on carbon non-LTE line formation using more advanced 3D atmsopheric simulation snapshots. The 3D non-LTE calculations in fact become feasible in a reasonable amount of time with this relatively simple atom.

The standard 217-level atom we use in this study is sufficiently complex to also include transitions commonly used as solar diagnostics. For example, our study confirms that the $538.0 \mathrm{~nm}$ line experiences small non-LTE effects $(<0.05 \mathrm{dex}$ in a solar-like model, as also found by Asplund et al. 2005a): solar observations could be used to test the non-LTE modelling. This feature becomes too weak below $[\mathrm{Fe} / \mathrm{H}] \sim-1$ and thus is not useful for determining $\mathrm{C}$ abundances at low metallicity. In this work we have instead paid particular attention to the high-excitation permitted C I lines around $910.0 \mathrm{~nm}$, which are

3 Because the fine-structure levels are not directly included in the whole problem. 

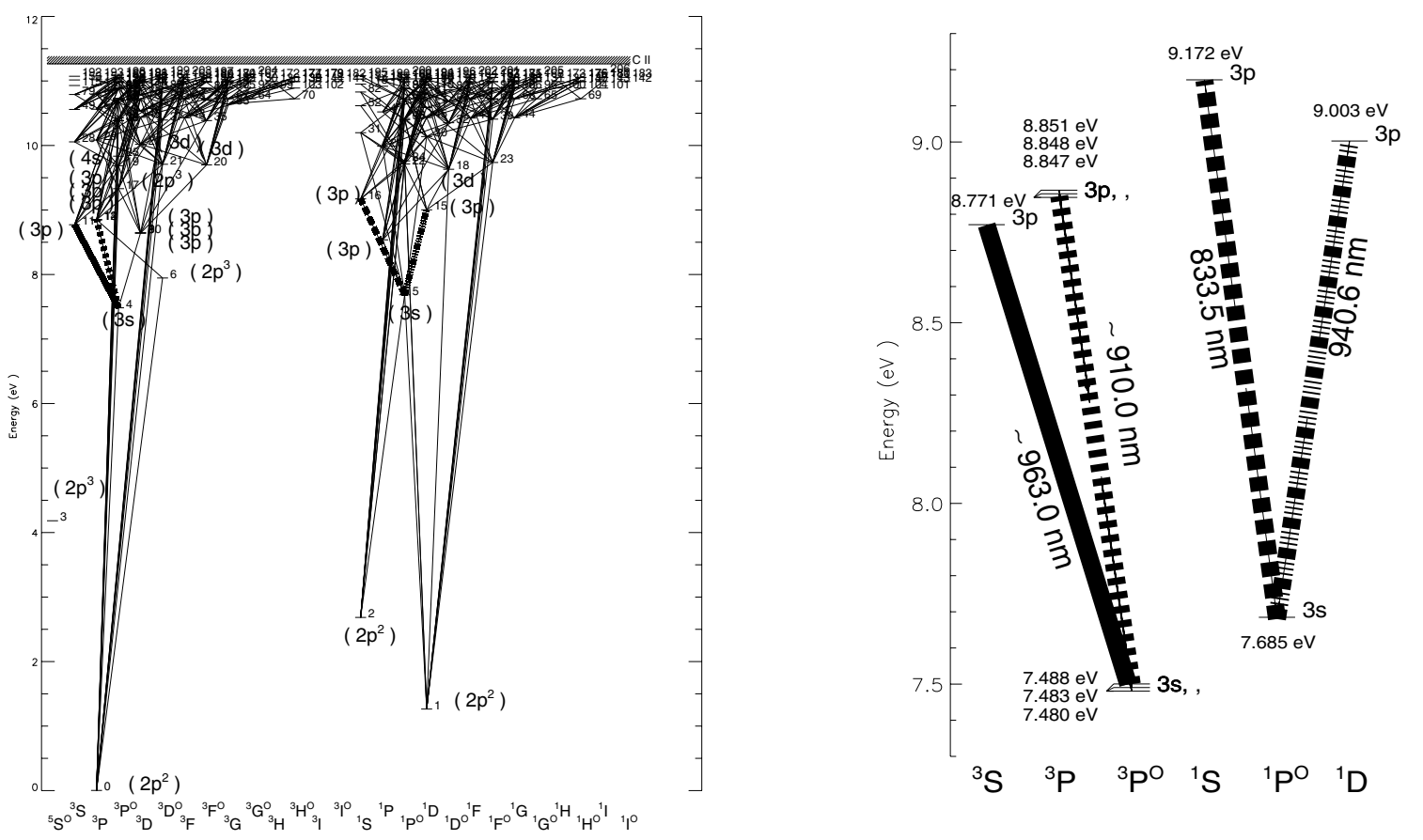

Fig. 1. Left panel: Grotrian term diagram for our 217-level (207 for C I, 9 for C II and one for C III) carbon model. For clarity, only the 453 bound-bound radiative transitions for $\mathrm{C}$ I are shown here. Right panel: Grotrian term diagram for the smaller carbon model atom used to compute the non-LTE abundance corrections for the Ci lines around 830.0-960.0 nm of particular interest here. The model includes $10 \mathrm{CI}$ levels and $1 \mathrm{C}$ II level. The bound-bound transitions in the triplet system generate from splitted levels in this model, producing three and six lines around 963.0 and $910.0 \mathrm{~nm}$ respectively.

Table 1. Details of the absorption lines included in the atomic model used after converged populations were obtained. A total of 11 transitions were treated in detail. Wavelength of absorption line, upper and lower level identification, excitation potential for lower level and oscillator strength are respectively given for each of the transitions included.

\begin{tabular}{cllcc}
\hline \hline $\begin{array}{c}\lambda \\
{[\mathrm{nm}]}\end{array}$ & $\mathrm{ID}_{l}$ & $\mathrm{ID}_{u}$ & $\begin{array}{c}\chi_{\text {exc }} \\
{[\mathrm{eV}]}\end{array}$ & $f_{\text {ik }}$ \\
\hline 960.3036 & $3 \mathrm{~s}^{3} \mathrm{P}_{0}^{\mathrm{o}}$ & $3 \mathrm{p}^{3} \mathrm{~S}$ & 7.480 & 0.127 \\
962.0781 & $3 \mathrm{~s}^{3} \mathrm{P}_{1}^{\mathrm{o}}$ & $3 \mathrm{p}^{3} \mathrm{~S}$ & 7.483 & 0.120 \\
965.8431 & $3 \mathrm{~s}^{3} \mathrm{P}_{2}^{\mathrm{o}}$ & $3 \mathrm{p}^{3} \mathrm{~S}$ & 7.488 & 0.105 \\
\hline 906.1433 & $3 \mathrm{~s}^{3} \mathrm{P}_{1}^{\mathrm{o}}$ & $2 \mathrm{p}^{3} \mathrm{P}_{2}$ & 7.483 & 0.150 \\
906.2492 & $3 \mathrm{~s}^{3} \mathrm{P}_{0}^{\mathrm{o}}$ & $2 \mathrm{p}^{3} \mathrm{P}_{1}$ & 7.480 & 0.350 \\
907.8288 & $3 \mathrm{~s}^{3} \mathrm{P}_{1}^{\mathrm{o}}$ & $2 \mathrm{p}^{3} \mathrm{P}_{1}$ & 7.483 & 0.088 \\
908.8515 & $3 \mathrm{~s}^{3} \mathrm{P}_{1}^{\mathrm{o}}$ & $2 \mathrm{p}^{3} \mathrm{P}_{0}$ & 7.483 & 0.124 \\
909.4834 & $3 \mathrm{~s}{ }^{3} \mathrm{P}_{2}^{\mathrm{o}}$ & $2 \mathrm{p}^{3} \mathrm{P}_{2}$ & 7.488 & 0.283 \\
911.1809 & $3 \mathrm{~s}^{3} \mathrm{P}_{2}^{\mathrm{o}}$ & $2 \mathrm{p}^{3} \mathrm{P}_{1}$ & 7.488 & 0.101 \\
\hline 940.5730 & $3 \mathrm{~s}{ }^{1} \mathrm{P}^{\mathrm{o}}$ & $3 \mathrm{p}{ }^{1} \mathrm{D}$ & 7.685 & 0.586 \\
\hline 833.5148 & $3 \mathrm{~s}{ }^{1} \mathrm{P}^{\mathrm{o}}$ & $3 \mathrm{p}{ }^{1} \mathrm{~S}$ & 7.685 & 0.110 \\
\hline
\end{tabular}

detectable down to low metallicity and have been used by Akerman et al. (2004). Our aim is to study departures from LTE for these lines, with a focus on the resulting $\mathrm{C}$ abundances in metal-poor halo stars once the non-LTE corrections are taken into account.

\subsection{Method}

The statistical equilibrium code MULTI ${ }^{4}$ (Carlsson 1986, 1992) was used to simultaneously solve the radiative transfer and rate equations for the C I non-LTE spectral-line formation. Complete

\footnotetext{
${ }^{4}$ Available at http://www. astro.uio.no/ matsc/mul22/
}

redistribution over Voigt line profile has been assumed. A grid of plane-parallel 1D MARCS (Gustafsson et al. 1975; Asplund et al. 1997, and subsequent updates) model atmospheres with varying $T_{\text {eff }}, \log g$ and $[\mathrm{Fe} / \mathrm{H}]$ has been employed in the calculations. The atmospheric parameter grid covers the range of late-type stars, comprising a total of 168 models with $4500 \leq$ $T_{\text {eff }} \leq 7000 \mathrm{~K}, 2.0 \leq \log g \leq 5.0[\mathrm{cgs}]$ and $-3 \leq[\mathrm{Fe} / \mathrm{H}] \leq 0$. In all cases, unless noted otherwise, the microturbulence velocity has been assumed to be $\xi=1.00 \mathrm{~km} \mathrm{~s}^{-1}$. An inherent assumption in our study is that the departures from LTE for carbon do not influence the given atmospheric structure. This is reasonable in the sense that carbon is not an important direct opacity nor a main electron contributor, so that the atmospheric opacity (and thus the equilibrium structure of the atmospheric model) will not change significantly even when large non-LTE corrections are found.

The standard solar $\mathrm{C}$ abundance adopted in our non-LTE radiative transfer calculations has been $\log \epsilon_{C_{\odot}}=8.40$, very close to the recently updated value (Asplund et al. 2005a,b). For each model atmosphere, the carbon abundance was varied to cover the range $-0.60 \leq[\mathrm{C} / \mathrm{Fe}] \leq+0.60$ in steps of 0.2 dex. We have repeated all calculations for three different choices of the scaling factor $S_{\mathrm{H}}$ for inelastic collisions $\left(S_{\mathrm{H}}=0,10^{-3}\right.$ and 1$)$.

\section{Results}

\subsection{Specific stars: Sun, Procyon, HD140283 and G64-12}

Initial tests were carried out for a few models representing specific stars (Sun, Procyon, HD 140283, G64-12), to check the effect of changes in different atomic properties and identify the main driving non-LTE effects for different stellar parameters. 
The high-excitation $\mathrm{CI}$ lines studied here are much stronger in Procyon $\left(T_{\mathrm{eff}}=6350 \mathrm{~K}, \log g=3.96,[\mathrm{Fe} / \mathrm{H}]=0.00\right.$, Allende Prieto et al. 2002) than in the Sun (5780/4.44/0.00), so that the higher line formation height in Procyon results in larger non-LTE effects. In the Sun, non-LTE abundance corrections vary between $\sim-0.10$ and $\sim-0.25$ dex for the lines of interest (when neglecting $\mathrm{H}$ collisions), while they rise to between $\sim-0.25$ and $\sim-0.50$ dex in Procyon. In both stars the drop of the line source function below the Planck function drives the departure from the LTE approximation. However, since the level populations deviate more from the LTE case when moving outwards in the atmosphere, in the case of Procyon the non-LTE abundance corrections are more severe.

The low-metallicity of HD 140283 (5690/3.67/-2.40) implies that the lines are weak and thus formed relatively deep in the atmosphere. Naively one would then expect the non-LTE effects to be quite small. Still, the non-LTE abundance corrections in this star vary between $\sim-0.20$ and $\sim-0.35$ dex, i.e. larger than in the Sun. This is mainly due to the lower $\log g$ and thus densities implying less efficient collisions. The lower $[\mathrm{Fe} / \mathrm{H}]$ also results in smaller electron pressures at a given optical depth and therefore again less important collisions. The level populations start to deviate relatively deep in the atmosphere of HD 140283, causing a line source function drop and an increased line opacity (because of the lower level of the transition getting overpopulated), both effects acting at the same time to strengthen the line. The non-LTE line formation in G64-12 (6511/4.39/3.20/) is similar to HD 140283: the lower $\log g$ and $[\mathrm{Fe} / \mathrm{H}]$ and higher $T_{\text {eff }}$ compared to the Sun all work in the same sense, thus implying even more pronounced non-LTE abundance corrections, between -0.2 and -0.45 dex, mainly due to an overpopulated lower atomic level (opacity effect making the line stronger than in LTE).

We have investigated the sensitivity of the non-LTE calculations to the adopted electron and hydrogen collisional crosssections. We ran calculations with a few different values for the parameter $S_{\mathrm{H}}$ regulating the $\mathrm{H}$ collisions efficiency. We found small abundance variations of the order of $\sim 0.1$ dex when changing $S_{\mathrm{H}}$ even by large factors, suggesting that the resulting nonLTE corrections are not particularly sensitive to the exact choice. This also guided us in what values to adopt $\left(S_{\mathrm{H}}=0,10^{-3}\right.$ and 1) when successively running full sets of calculations for the whole grid of stellar models described below. We checked the effect of electron collisions by artificially decreasing their cross-sections by a factor of 10 . Also in this case, the effects were small and non-negligible only at low metallicity where they reach $\sim \pm 0.03$ dex at most.

We finally tested the influence on the non-LTE corrections when increasing the microturbulence velocity from 1.0 to $1.5 \mathrm{~km} \mathrm{~s}^{-1}$. A higher value for this parameter, introduced in 1D atmospheric models as an effective (though not perfect) substitute for convective motions, is expected to affect mostly the stronger saturated lines, since the atoms will absorb photons at slightly shifted wavelengths (where the flux is higher) compared to the line center. The stronger lines will thus be strengthened and lower abundances should be obtained from them. By appropriately adjusting the microturbulence parameter, it is possible to remove trends of derived abundances with excitation potential or line strength which appear when velocity fields are ignored. As expected, the lines' equivalent widths (both LTE and nonLTE) increase slightly, by only a few $m \AA$ when increasing the microturbulence to $\xi=1.5 \mathrm{~km} \mathrm{~s}^{-1}$. However, the resulting nonLTE abundance corrections experience insignificant variations, decreasing by $<0.01$ dex.

\subsection{Non-LTE effects}

The departure coefficient $\beta$ for an atomic level is defined as $\beta=N / N_{\text {LTE }}$, where $N$ and $N_{\text {LTE }}$ are the atomic populations in non-LTE and LTE respectively, for the level considered. The ratio of departure coefficients for different atomic levels enters the line source function ${ }^{5} S_{v}^{1}$, so that (when neglecting stimulated emission, as should be possible for the lines considered here) identical values of $\beta_{\mathrm{u}}$ and $\beta_{\mathrm{l}}$ (for the lower and upper level of a transition), result in a Planckian line source function. This means that even when both levels in a transition have populations that depart considerably from the Boltzmann expectation, it is possible that the net effect is a line source function close to LTE, as long as the departures are the same for the two levels. However, the effect of line opacity must also be considered when the lower level population increases or decreases with respect to the LTE approximation, since it will affect the depth of line formation.

The leftmost panels in Fig. 2 show, for a representative case $\left(T_{\text {eff }}=6000 \mathrm{~K}, \log g=4.50\right.$ and collisional efficiency set to $S_{\mathrm{H}}=10^{-3}$ ), the typical departure coefficients $\beta$ for the upper and lower levels of the $C$ I line at $\sim 910.0 \mathrm{~nm}$. The figure highlights how at solar metallicity the dominant non-LTE effect is the line source function dropping below the Planck function, mostly due to the decreased population of the upper level compared to LTE, while at low metallicity the line source function is close to LTE, in particular at the much deeper formation layers in such stars, i.e. closer to the continuum optical depth; the location of $\tau_{\text {continuum }}=1$ and $\tau_{\text {line center }}=1$ is marked in the figure. The non-LTE effects are found to be large even for the most metal-poor stars in our grid. To explain this, we used the contribution functions defined by Magain (1986) to evaluate the contribution of the different atmospheric layers to the formation of the spectral-line depression. Especially at low metallicity, we found contributions from layers much higher up than the canonical line optical depth unity to be important. The non-LTE line strengthening effect can then be explained in such case, in terms of increased line opacity: the lower level of the transition gets overpopulated with respect to LTE in those layers, as seen in the departure coefficients plot for this metallicity (Fig. 2), causing the still large non-LTE corrections found in this case. In stars with $[\mathrm{Fe} / \mathrm{H}] \sim-1$, the two effects just described (line source function drop and line opacity increase) work in unison, resulting in large non-LTE effects (see Fig. 6).

Figures 3 and 4 show results from "multi-MULTI" runs. In such calculations, all 650 radiative transitions and a subset of all collisional transitions (all collisional rates between the lowest 26 atomic levels) were perturbed in turn by a factor of 2 to study the impact on the $\mathrm{C}$ I lines of main interest here. The runs were carried out for a star with $T_{\text {eff }}=6000 \mathrm{~K}$ and $\log g=4.50$ and metallicities from solar to $[\mathrm{Fe} / \mathrm{H}]=-3$. Since for every transition that is changed in the atomic model a full MULTI run has to be performed, the multi-MULTI runs are very time-consuming. We refer to Carlsson et al. (1994) for a detailed description of how such runs are carried out.

Figure 3 confirms that for the $\sim 910.0 \mathrm{~nm} \mathrm{C}$ I feature, the driving non-LTE effect at solar metallicity is the line source function drop. The $\sim 166.0 \mathrm{~nm}$ resonance line from the ground state $\left(2 \mathrm{p}^{2} 3 \mathrm{P}-3 \mathrm{~s}{ }^{3} \mathrm{P}^{\mathrm{o}}\right)$ is an extremely optically thick transition and cannot drain the population of its upper level (which is the lower level of the $\sim 910.0 \mathrm{~nm}$ transition), which will thus stay

\footnotetext{
5 More explicitly, assuming complete redistribution and in the Wien regime, the ratio of the line source function to the Planck function can be expressed in terms of the ratio of departure coefficients for the upper and lower levels of a transition, as $S_{v}^{1} / B_{v} \approx \beta_{\mathrm{u}} / \beta_{\mathrm{l}}$.
} 

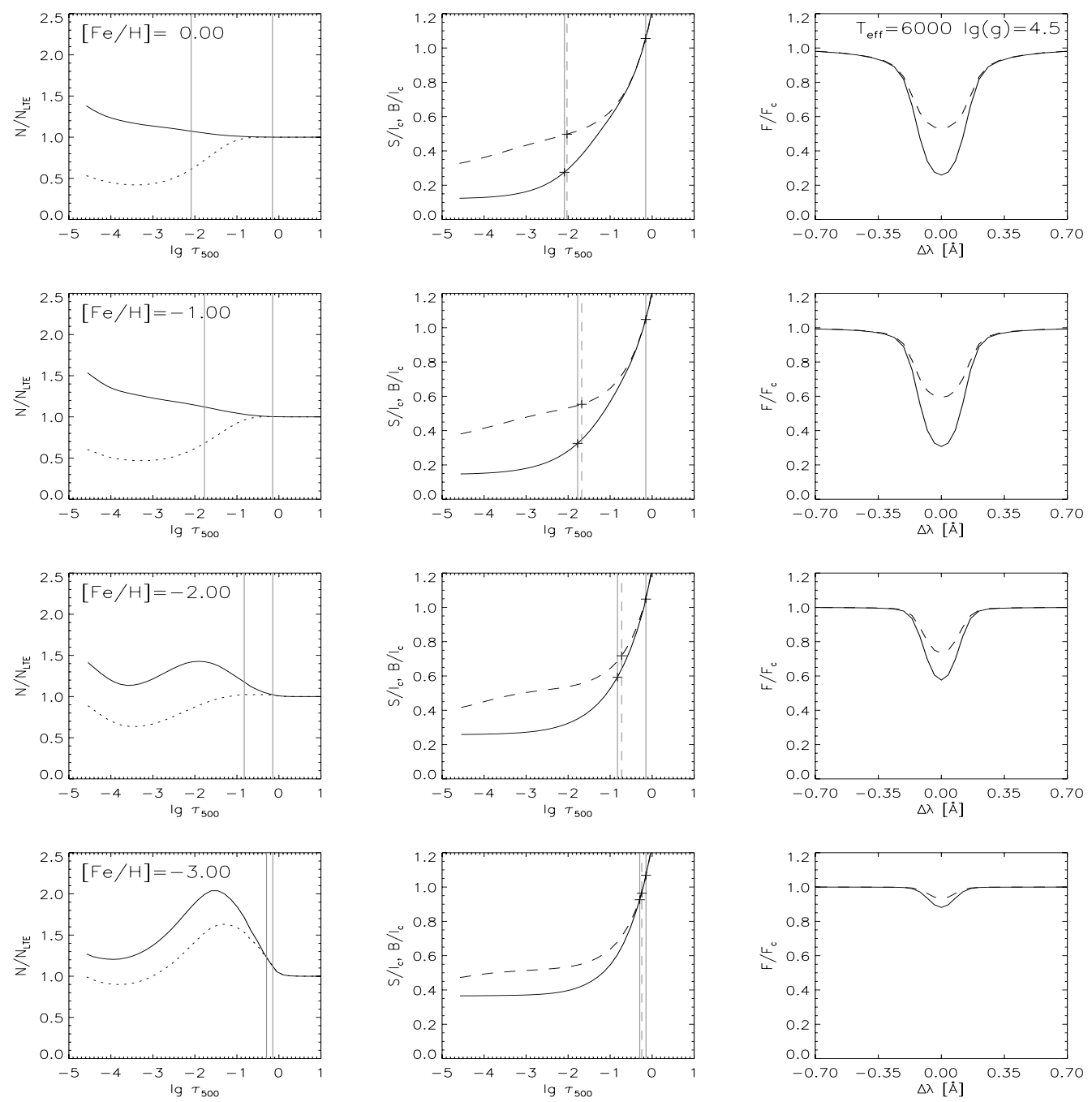

Fig. 2. Departure coefficients from LTE (left panels), line source and Planck function comparison (middle panels) and resulting line profile (right panels) for the $\mathrm{C}$ I line at $\sim 910.0 \mathrm{~nm}$ shown for different $[\mathrm{Fe} / \mathrm{H}]$ for runs with our larger atomic model. The atmospheric models all have $T_{\text {eff }}=6000 \mathrm{~K}, \log g=4.50[\mathrm{cgs}]$ and $[\mathrm{C} / \mathrm{Fe}]=0$, with $[\mathrm{Fe} / \mathrm{H}]$ going from 0.0 (top) to -3.0 (bottom). The hydrogen collision efficiency has been set to $S_{\mathrm{H}}=10^{-3}$. For the departure coefficients the solid curve represents the lower level and the dotted one the upper level for the transition of interest. In the middle panels the line source function (solid line) and Planck function (dashed line) are normalized to the continuum intensity. Location of $\tau_{\text {continuum }}=1$ (solid line at $\log _{10} \tau_{500} \sim 0$ ) and $\tau_{\text {line center }}=1$ (in LTE and non-LTE, dashed and solid line respectively) are shown by vertical lines in the left and middle panels. In all cases, the non-LTE line profile (solid line) is stronger than in LTE (dashed line).

close to LTE $\left(\beta_{1} \sim 1\right)$ where the line is formed due to sufficiently strong coupling to the ground state through electron collisions (see Fig. 3). The upper level of the transition of interest gets instead underpopulated in non-LTE $\left(\beta_{\mathrm{u}}=N / N_{\mathrm{LTE}}<1\right)$, mainly through photon losses in the line itself, see Fig. 5. Since the ratio $\beta_{\mathrm{u}} / \beta_{1}$ determines the line source function, this explains why the latter drops below the Planck function $\left(S_{v}^{1}<B_{v}\right)$ around solar metallicity (Fig. 2), causing the line strenghtening effect. The effect of collisions (mainly in the triplet system to which the line studied belongs) is instead to weaken the line, tending to restore the LTE populations, however the line source function drop still dominates, driving the non-LTE line strengthening. At this metallicity, the $\sim 910.0 \mathrm{~nm} \mathrm{C}$ I line will therefore be most sensitive to processes that affect the upper level population, thus changing the line source function. There is very little or no coupling with the singlet system at this metallicity, so that only a small influence from collisions in the singlet system is seen. Photoionization is not important either.
For metal-poor stars, the line source function is close to LTE in the deep layers where the line is formed. Indeed, even though both levels tend to get overpopulated with respect to LTE, the ratio of their departure coefficients stays close to the LTE value $\left(\beta_{\mathrm{u}} / \beta_{1} \sim 1\right)$ where the line is formed ${ }^{6}$. However, the onset of significant departures from LTE in the level population is now shifted to deeper atmospheric layers than for the solar case. Moreover, the overpopulation of the lower level in these layers makes the line form further out in the atmosphere (where

6 It is important to remember that, as mentioned, these weak lines form with a significant contribution from a tail of atmospheric layers located much higher up than one would expect in the simple EddingtonBarbier approach, as we discovered by looking at their contribution functions (Magain 1986). For example, at $T_{\text {eff }}=6000 \mathrm{~K}, \log g=4.50$, $[\mathrm{Fe} / \mathrm{H}]=-3$, the average height of formation actually moves out to $\log _{10} \tau_{500} \sim-0.7$, with a significant contribution to the absorption profile still coming from layers as high up as $\log _{10} \tau_{500} \sim-2$. Also note the steep increase of the level population at low metallicity, as one moves outward in the atmospheric model (Fig. 2). 

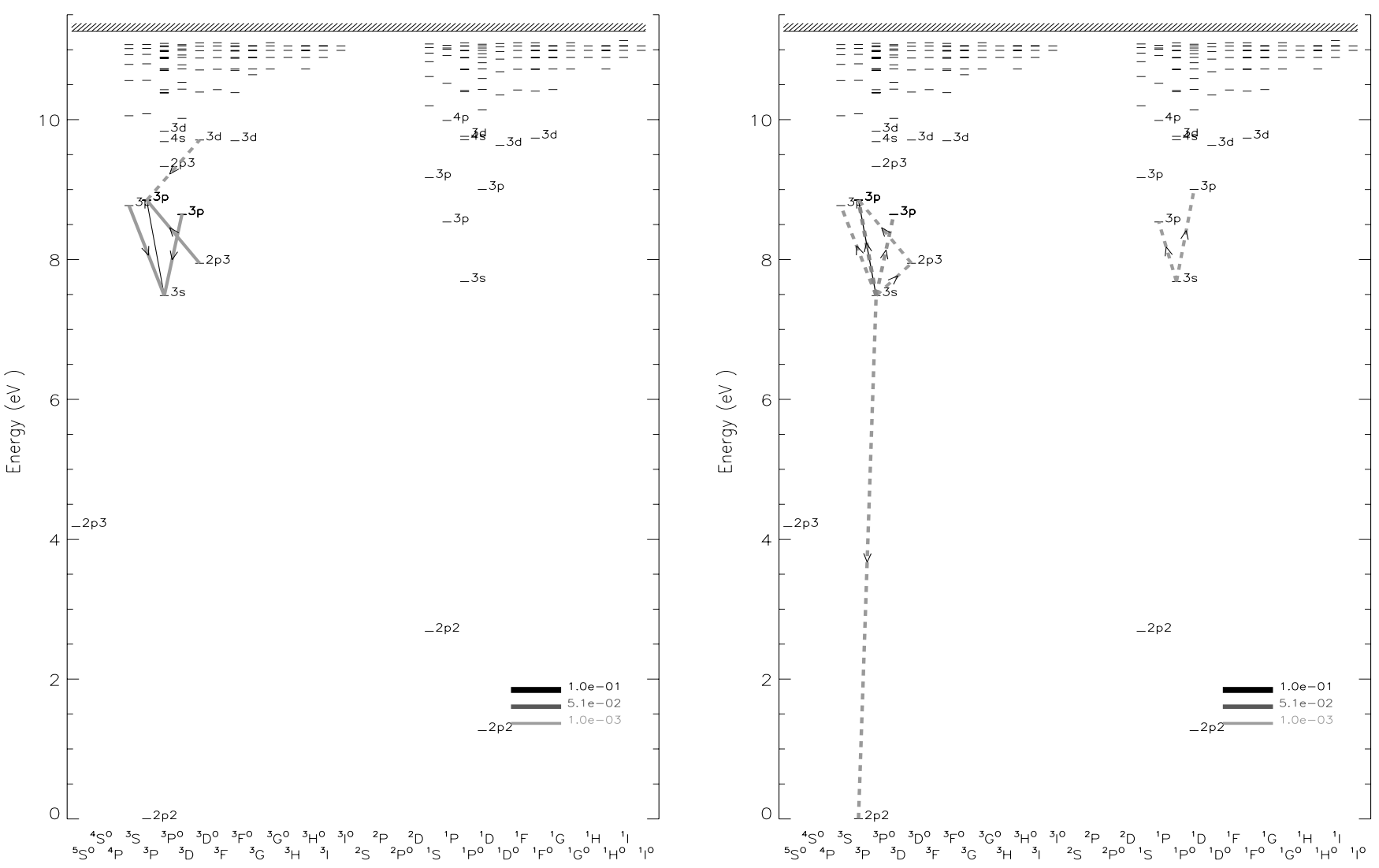

Fig. 3. Results from multi-MULTI runs for a star with $T_{\text {eff }}=6000 \mathrm{~K}$ and $\log g=4.50$ and solar metallicity are shown for the C I line around $910.0 \mathrm{~nm}$. The effect on the line strength of multiplying each of the different rates for radiative transitions in turn by a factor of 2 is shown in the left panel. The right panel shows the effect when varying the collisional rates individually by the same amount. Solid lines indicate positive contribution (strengthening), dashed lines mean negative contribution (weakening) from that particular transition to the strength of the line studied. The thickness indicates the relative change in the non-LTE equivalent width $\left(W^{\text {pert }}-W^{\text {ref }}\right) / W^{\text {ref }}$ between the perturbed and reference case, as in the figure legend, while the arrows indicate the net flow (evaluated at $\tau_{\text {line center }}=1.0$ ) for the transitions.

deviations in the level populations are more dramatic) than in LTE, thus they are stronger than in that case. This opacity effect causes the line strengthening to be sensitive to processes affecting the lower level population. Even though electron collisional coupling to the ground state still tends to restore LTE population in the lower level of the $\sim 910.0 \mathrm{~nm}$ transition also at low metallicity, the latter level now gets severely overpopulated across the atmosphere, due to the fact that the total sum of the net radiative rates dominates with respect to collisional ones for this transition (see Fig. 5) and that it cannot depopulate efficiently and will act as a meta-stable level in detailed balance with the ground state. Moreover, in the low-metallicity case, the line strength is influenced by processes in the singlet system much more than at solar metallicity, i.e. the coupling between the triplet (to which the lines studied belong) and singlet systems becomes important (Fig. 4) and starts to influence the line strength more and in a more complex way, both through radiative rates and collisional transitions. So, while at solar metallicity the magnitude of the non-LTE effects is mainly governed by collisions and radiative transitions between levels in the triplet system itself, with decreasing metallicity both the intersystem (triplet-singlet) collisions and the processes in the singlet system (collisions as well as radiation) gain importance with respect to processes in the triplet system. Electron collisions, especially those between levels with similar energy, activate the coupling with processes in the singlet system and thus for example play an important role in determining the population balance of the lower level of the transition of interest. The use of accurate collisional rates is therefore important. Even though at low metallicity the lines studied are weak, thus formed deeper in than for solar metallicity (Fig. 2), and one could naively assume the non-LTE effects to be small, it is the relative strengthening of the line with respect to LTE that justifies the still large non-LTE corrections we found for low-metallicity stars. The main non-LTE effect as one moves to low metallicity is thus an increased line opacity arising from an overpopulated lower level, with the contribution coming from the line source function effect still present but becoming less important with respect to the solar metallicity, because both the lower and upper levels of the transition are now similarly overpopulated in the line-forming layers.

Note that in Figs. 3 and 4, the upward or downward direction of the rates (represented by arrows) is evaluated at $\tau_{\text {line center }}=$ 1.0. In reality such rates can have a complicated behaviour with atmospheric depth. For example, for the left panel (solar metallicity, radiative case) in Fig. 3, the rate for the transition from level $2 \mathrm{~s} 2 \mathrm{p}^{3} 3 \mathrm{D}^{\mathrm{o}}$ at $\sim 7.95 \mathrm{eV}$ to $2 \mathrm{~s}^{2} 2 \mathrm{p} 3 \mathrm{p} 3 \mathrm{P}_{2}$ at $\sim 8.85 \mathrm{eV}$ (i.e. from level 6 to 14, in the notation adopted in Fig. 1) is upward, but it is found to become downward just one depth-point below $\tau_{\text {line center }}=1.0$. The net result of this is that the upper level population density for the $\mathrm{C}$ I transition around $910.0 \mathrm{~nm}$ actually decreases when increasing this f-value, which explains why such line becomes stronger (a line source function effect, because $\beta_{\mathrm{u}} / \beta_{1}$ becomes less than unity, thus making $S_{v}^{1}<B_{v}$ ).

In Fig. 5 we plot the net radiative and collisional rates for the transitions that most influence the lower and upper level of the transition at $\sim 910.0 \mathrm{~nm}$. One for example sees that at 


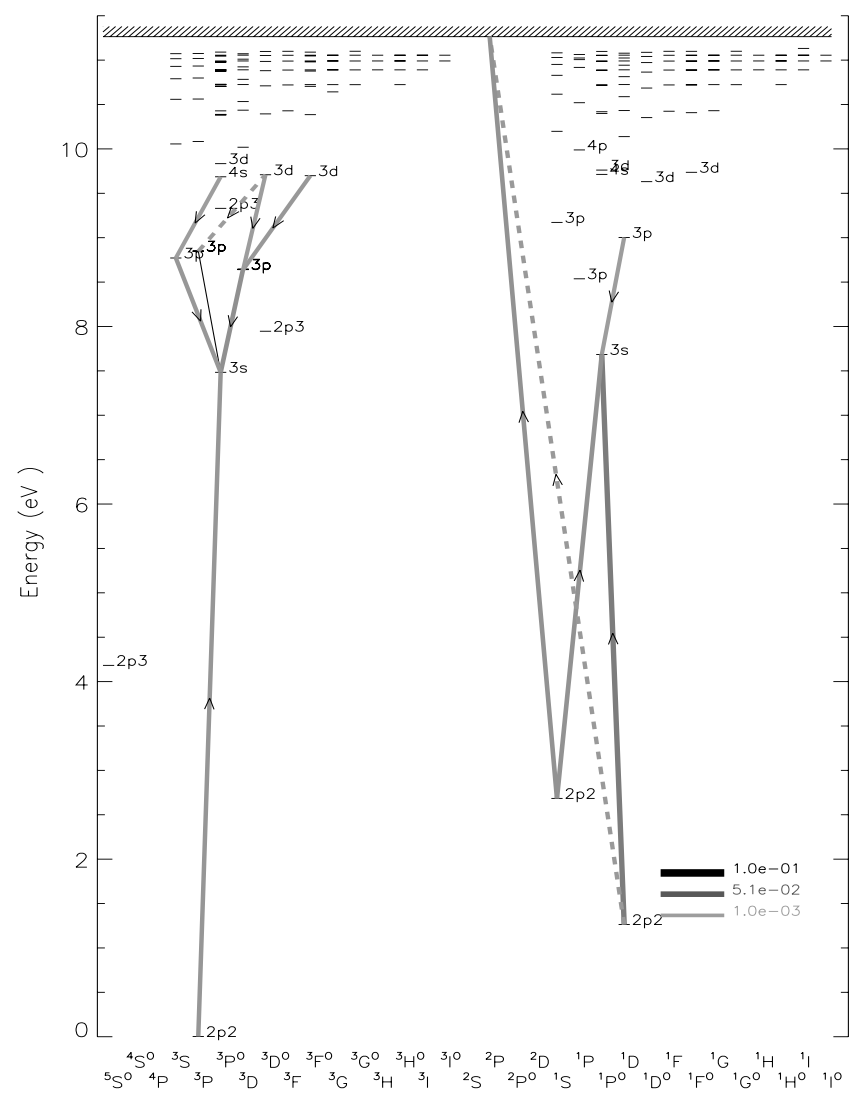

Fig. 4. Same as Fig. 3, but for $[\mathrm{Fe} / \mathrm{H}]=-3$.

solar metallicity the population balance of the upper level ( $3 p$ ${ }^{3} \mathrm{P}_{2}$, labelled as level 14 in the figure, as per notation adopted here of increasing level number with increasing energy, with level 0 being the ground state), shown in the middle left plot, is set by radiative losses to the lower level of the transition itself $\left(3 \mathrm{~s}{ }^{3} \mathrm{P}^{\mathrm{o}}\right)$ balanced by collisions from this same level and from level $3 p{ }^{3} \mathrm{P}_{1}$ which has energy close to that of the upper level. The line will thus get stronger if any of the levels feeding into the upper level get underpopulated, conversely it will get weaker if we increase the population of any of these. This picture confirms what is seen in Fig. 3 and described above, namely that collisions generally have a weakening effect on the line, by tending to restore LTE populations. The sum of all net radiative rates is predominant in magnitude with respect to that of collisional rates, only slightly in the solar-metallicity case, while it strongly dominates at low metallicity (bottom panels of Fig. 5). The effect is to drive the balance away from LTE, with a decreased upper level population in the solar metallicity case mainly due to photon loss in the line itself (the lower level population remaining close to LTE due to collisional transitions out of that level which tend to mitigate non-LTE effects by contrasting the effect of radiative transtions from higher levels) and an increased population for both levels at low metallicity (the population flow from higher levels into the lower level of the transition causing its overpopulation and thus driving the departure from LTE). As line transitions turn out to be important, in different ways, both at solar and low metallicity, our choice of including as many levels and accurate radiative rates in the atomic model employed (see Sect. 2.2), making it more complete than those used in some previous studies by other authors (e.g. Stürenburg \& Holweger 1990; Rentzsch-Holm 1996a,b), is particularly well suited.

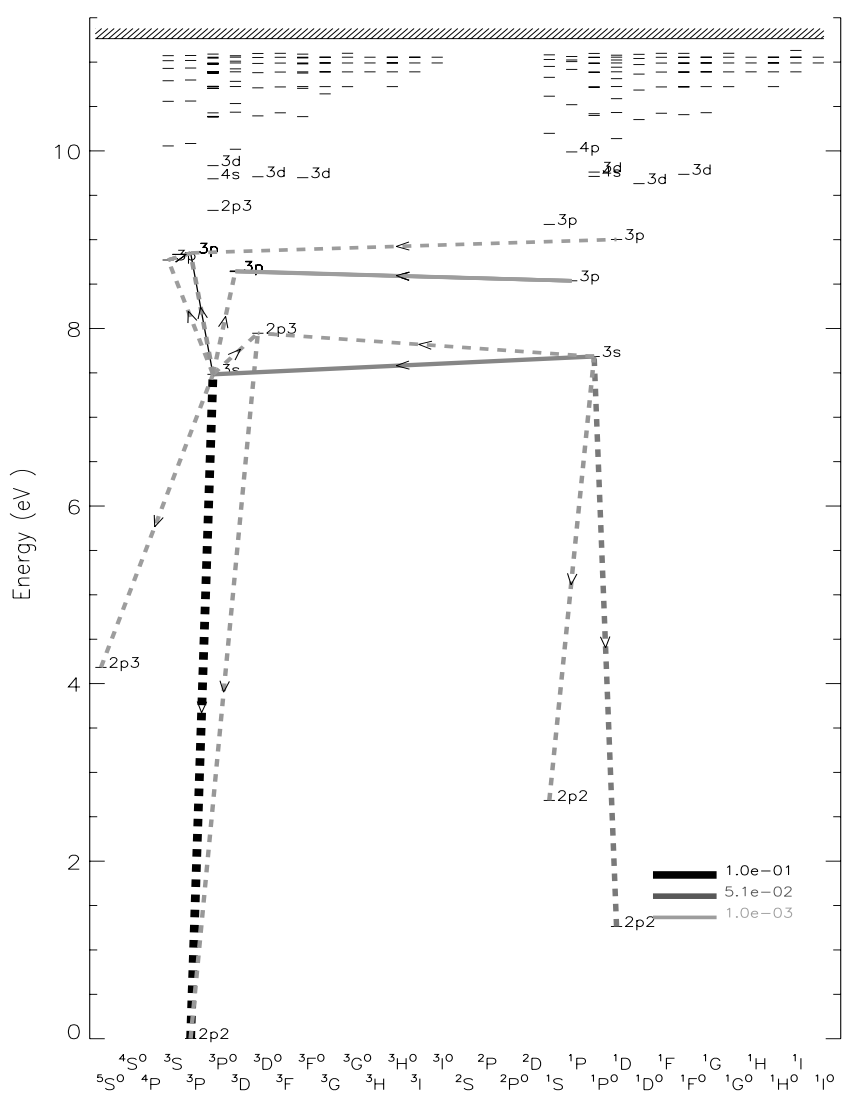

Our results underline that the non-LTE effects for the carbon lines considered have different causes in different stars. It is thus paramount to carefully evaluate such effects on a star-to-star basis, in stellar abundance studies.

\subsection{Non-LTE abundance corrections}

The C I non-LTE line strength for the parameter grid explored is generally larger than in LTE. The non-LTE abundance corrections reach down to as much as -0.8 dex and more for particular combinations of atmospheric parameters (notably at $T_{\text {eff }} \geq 6000 \mathrm{~K}, \log g=2.00,[\mathrm{Fe} / \mathrm{H}]=-1.00$, with corrections of up to $\sim-1.0 \mathrm{dex}$ ). For each set of atmospheric parameters, the resulting non-LTE abundance corrections become more severe for stronger lines, similarly to what is found for the solar case (Asplund et al. 2005a). This can be explained intuitively because stronger lines will be formed higher up in the atmosphere where departures from LTE in the level populations are often more significant.

Several studies (e.g. Kaulakys 1985; Fleck et al. 1991; Belyaev et al. 1999; Belyaev \& Barklem 2003) now seem to indicate that Drawin's classical recipe (1968) might overestimate the efficiency of $\mathrm{H}$ collisions by about three orders of magnitude. In view of the corresponding uncertainty of our final results due to this treatment of the excitation and ionization by $\mathrm{H}$ collisions, we have chosen to perform full sets of calculations for the whole parameter grid, applying Drawin's formula with different choices of the scaling factor $S_{\mathrm{H}}$ for separate runs, to test how much this affects our derived non-LTE abundance corrections. We have adopted $S_{\mathrm{H}}=0,10^{-3}$ and 1 respectively and have found a relatively small sensitivity of the resulting non-LTE 


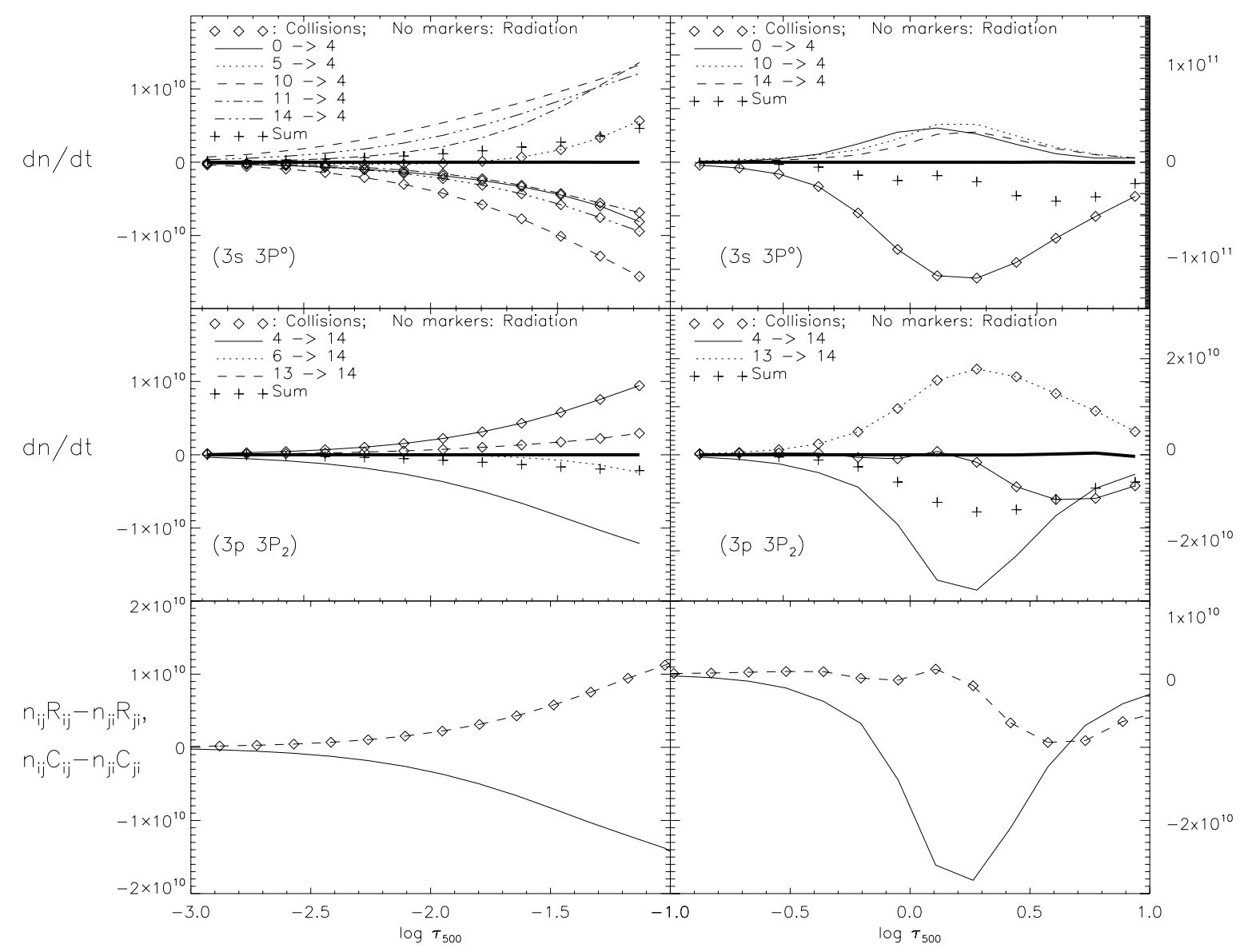

Fig. 5. The net rates for radiative (no markers) and collisional (with markers) transitions are shown in the upper left plot for the lower level of the transition at $\sim 910.0 \mathrm{~nm}$ in a model with $T_{\text {eff }}=6000 \mathrm{~K}, \log g=4.50$ and solar metallicity. Only the rates for the transitions important for the level balance are shown, marked with different symbols. Their sum is also marked with crosses and confirms that the rest of the levels play a minor role here. The horizontal line at $\mathrm{d} n / \mathrm{d} t=0$ marks the expectation that the total sum of all rates is equal to zero. The levels in the legends are numbered accordingly to our atomic model (see Fig. 1), i.e. increasing with excitation potential. The middle left plot similarly shows the rates for the upper level of the transition, while the bottom left plot shows the total sum of the net radiative and collisional rates respectively (positive $=$ upwards). The plots on the right show the same quantities, but for a model with $[\mathrm{Fe} / \mathrm{H}]=-3$. Note the different scale for the vertical axes in the plots with different metallicity.

corrections to the value of this parameter. The non-LTE abundance corrections obtained for all stellar models in our grid, from calculations adopting the two extreme choices of $S_{\mathrm{H}}$ are shown in Fig. 6 for the carbon absorption line at $909.4834 \mathrm{~nm}$. The plot refers to results obtained when adopting $[\mathrm{C} / \mathrm{Fe}]=+0.4$, which is the typical LTE value for the most metal-poor halo stars in the Akerman et al. (2004) sample. As expected, we find slightly larger non-LTE abundance corrections when setting a low efficiency for $\mathrm{H}$ collisions. In all cases, stars in the higher temperature, lower gravity range appear to be particularly affected, with very large non-LTE effects. The corrections peak around a metallicity $[\mathrm{Fe} / \mathrm{H}] \sim-1$. As seen in the figure, when neglecting inelastic H collisions or assuming Drawin's recipe (1968) for their treatment, the non-LTE corrections only vary marginally $(\lesssim 0.1$ dex difference between corrections obtained in the two cases). Since our choices of $S_{\mathrm{H}}$ should bracket the true values, it is reassuring that the corresponding uncertainty in the non-LTE corrections is small.

At the lowest metallicity, the non-LTE effects are still important, with $\Delta \log \epsilon_{\mathrm{C}} \sim-0.35$ dex for typical halo stars parameters $\left(T_{\text {eff }} \simeq 6000 \mathrm{~K}, \log g=4.00\right)$ and a choice of $S_{\mathrm{H}}=10^{-3}$. When we instead adopt Drawin's recipe as is (parameter regulating collisions with H I set to $S_{\mathrm{H}}=1$ ), the resulting non-LTE corrections remain important and are only slightly smaller (still amounting to $\sim-0.25$ dex at the lowest metallicity for typical halo stars parameters) than the previous case. Figure 7 shows the large non-LTE corrections obtained for a representative set of atmospheric parameters $\left(T_{\text {eff }}=6000 \mathrm{~K}\right.$ and $\left.\log g=4.00\right)$. They indeed reach down to $\sim-0.45 \mathrm{dex}$ at $[\mathrm{Fe} / \mathrm{H}]=-1$ and are still very substantial $\left(\Delta \log \epsilon_{\mathrm{C}} \sim-0.35 \mathrm{dex}\right)$ even for weak lines at $[\mathrm{Fe} / \mathrm{H}]=-3$. Note that in these plots, only corrections obtained with $[\mathrm{C} / \mathrm{Fe}]=+0.00$ are shown. For larger $[\mathrm{C} / \mathrm{Fe}]$ the line formation is shifted outwards and hence the non-LTE corrections become even more severe.

Our analysis aims at removing (some of the) potential systematic errors in stellar abundance analyses. With this in mind, we provide an IDL routine ${ }^{7}$ that interpolates our predicted nonLTE abundance corrections to give the result for arbitrary stellar parameters and carbon abundances within our grid.

\footnotetext{
7 Available via FTP from:

ftp://ftp.mso.anu.edu.au/pub/damian/corr_nlte/c/
} 

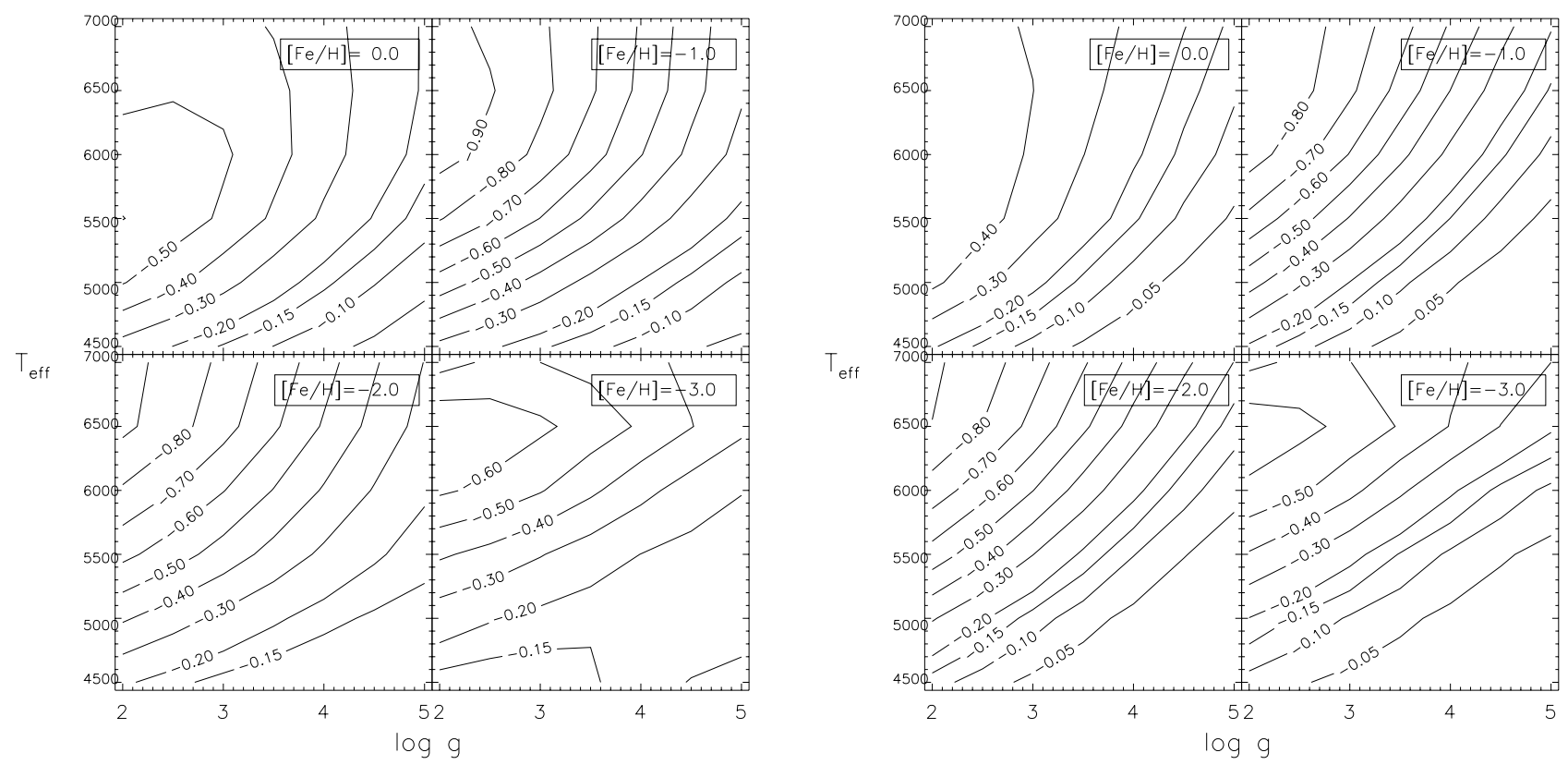

Fig. 6. Non-LTE abundance corrections over the grid of temperatures and gravities explored, with varying metallicity (as indicated in the insets), for the neutral carbon absorption line at $909.4834 \mathrm{~nm}$ (used e.g. by Akerman et al. 2004, as one of the abundance indicators for their determination of $\mathrm{C}$ in halo stars). The corrections refer to the case with $[\mathrm{C} / \mathrm{Fe}]_{\mathrm{LTE}}=+0.4$, typical of metal-poor halo stars and have been obtained from calculations with fine splitting included in the atomic model. The four leftmost panels show the corrections we obtained when neglecting hydrogen collisions, while the rightmost panels represent the results with $S_{\mathrm{H}}=1$. Large non-LTE corrections are present at high temperature and low gravity, in the various cases.

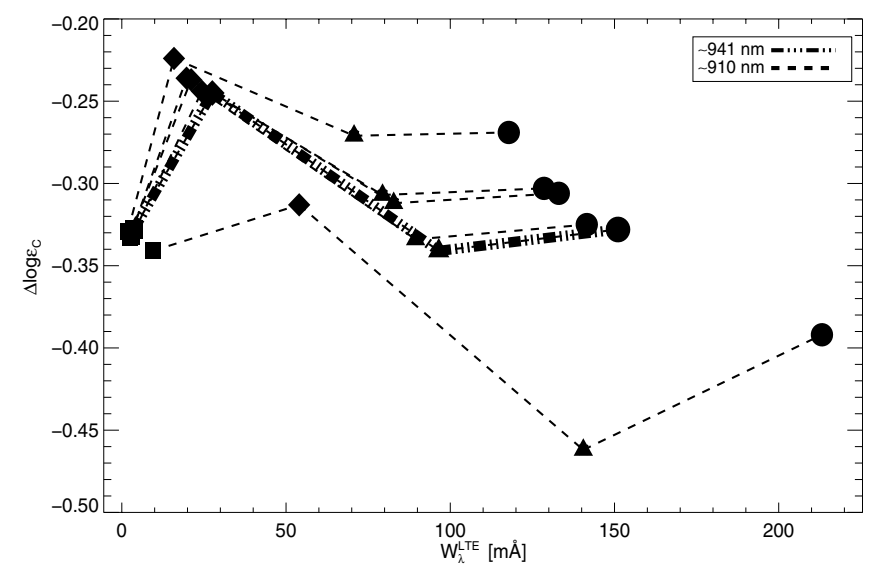

Fig. 7. The non-LTE abundance corrections are plotted versus line strength for the CI lines around 941 and $910 \mathrm{~nm}$. The models have $T_{\text {eff }}=6000 \mathrm{~K}, \log g=4.0$ and $[\mathrm{Fe} / \mathrm{H}]=-3,-2,-1$ and 0 (for which different symbols are used in the figure: filled squares, diamonds, triangles and circles respectively). Here $[\mathrm{C} / \mathrm{Fe}]=0$ and $S_{\mathrm{H}}=10^{-3}$ have been assumed. For each absorption line, the non-LTE corrections at different metallicities are connected to underline the effect of the metallicity change.

\section{Comparison with previous non-LTE studies}

Only a small number of previous studies have investigated the non-LTE formation of carbon lines in late-type stars (Stürenburg \& Holweger 1990; Takeda 1994; Paunzen et al. 1999; Takeda \& Honda 2005; Asplund et al. 2005a). With the exception of Takeda \& Honda (2005), these have all been restricted to a few specific stars rather than covering a large parameter space as we did in our study.
To determine the carbon abundances of their sample of disk stars, Takeda \& Honda (2005) use the C I 505.2/538.0 nm permitted lines. These abundance indicators were also used by Asplund et al. (2005a) for a determination of the solar photospheric carbon abundance. In both cases, very small non-LTE effects were found for such lines. We confirm that these features have small negative non-LTE abundance corrections ( $\Delta \log \epsilon_{\mathrm{C}}$ no larger than $\sim-0.05$ dex for solar-type stars). To reanalyse the metal-poor halo stars available in the literature, Takeda \& Honda carried out non-LTE calculations down to $[\mathrm{Fe} / \mathrm{H}]=-4$ in their work, to determine the departure coefficients for the levels relevant to the same high-excitation features we also study in detail. They used a carbon model atom with 129 C I levels (Takeda 1992) for an extensive parameter grid similar to ours but adopting $[\mathrm{C} / \mathrm{Fe}]=0$ and \pm 0.3 dex only in the calculations. For the collisional rates due to neutral hydrogen atoms, they also adopted Drawin's formula in the generalized version by Steenbock \& Holweger (1984), with the default choice $S_{\mathrm{H}}=1$ for all calculations. We find a generally good agreement between the two non-LTE studies. However for a few models with high $T_{\text {eff }}$ and low $\log g$ and $[\mathrm{Fe} / \mathrm{H}]$ our non-LTE abundance corrections, although very large $\left(\Delta \log \epsilon_{\mathrm{C}} \simeq-0.8 \mathrm{dex}\right)$, are not as severe as claimed by Takeda \& Honda (see our Fig. 8).

We suspect that the reason for this difference is an extrapolation error in the work by Takeda \& Honda, possibly due to their use of only $[\mathrm{C} / \mathrm{Fe}]=0$ and \pm 0.3 . For the halo stars available in the literature, they also find relatively large $\left(\left|\Delta \log \epsilon_{\mathrm{C}}\right| \lesssim\right.$ 0.3 dex), negative, metallicity-dependent non-LTE corrections below $[\mathrm{Fe} / \mathrm{H}]=-2$, those values however being still $\sim 0.05$ dex smaller than what we obtain at such low metallicities.

Stürenburg \& Holweger (1990) found that C non-LTE abundance corrections in the Sun are typically small $(\$-0.05$ dex on average when adopting a scaling factor of $1 / 3$ to Drawin's formula for the $\mathrm{H}$ collisions). For the lines we also study here, they 


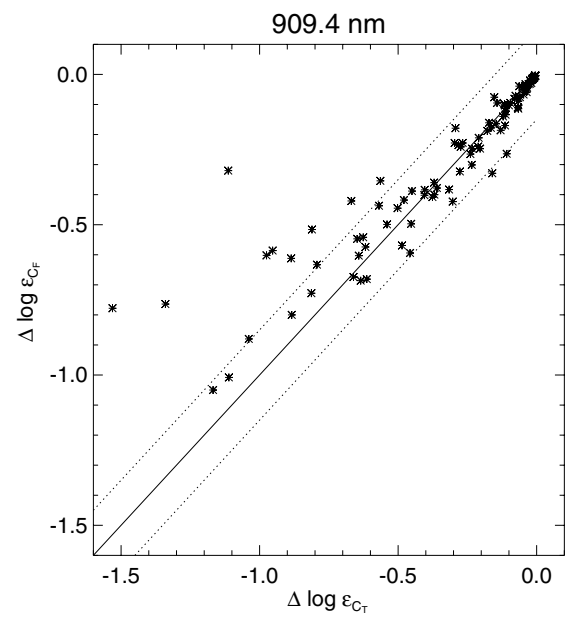

Fig. 8. The non-LTE abundance corrections we found in this work (vertical axis scale) for the $909.4834 \mathrm{~nm}$ absorption line in a range of atmospheric models are compared to the corresponding results in Takeda \& Honda (2005), for the case $S_{\mathrm{H}}=1$. The solid line marks the one to one relation, while the dotted lines include a region where the difference between the corrections in the two studies is limited to $\leq 0.15 \mathrm{dex}$.

explained the non-LTE effects in the solar photosphere mainly in terms of lower level overpopulation, causing the line absorption coefficient to exceed the LTE value. We underline that the driving effect at solar metallicity is instead that of the line source function dropping below the Planck function, i.e. both the lower and upper levels of the transition need to be considered. The upper level underpopulation is actually more significant than the lower level (slight) overpopulation in the line-forming region, driving the line source function drop in Sun-like stars (see our Fig. 2). In any case, a detailed comparison between the results for the high-excitation C I lines of interest shows a very good agreement, the non-LTE abundance corrections amounting to $\sim-0.1$ dex for those features in both studies.

Using the same model atom as Stürenburg \& Holweger, Tomkin et al. (1992) find comparably small non-LTE effects for the $\mathrm{C} \mathrm{I} \sim 910.0 \mathrm{~nm}$ lines $\left(\Delta \log \epsilon_{\mathrm{C}} \lesssim-0.15 \mathrm{dex}\right)$ also in metalpoor halo stars. However, they adopted very large efficiencies for the $\mathrm{H}$ collisions, in order to reproduce the observed line strengths using the high solar carbon abundance expected at the time. As explained, evidence is now accumulating that such high values for $\mathrm{H}$ collisions might be unrealistic by several orders of magnitude, so that the non-LTE corrections for the high-excitation C I features of interest should be expected to be in reality more significant than in that study.

Using an extended version of the atomic model by Stürenburg \& Holweger, Rentzsch-Holm (1996a,b) investigated carbon non-LTE abundance corrections in A-type and related stars. A detailed comparison with that work is unfortunately not possible, since the two calculations only overlap for our highest temperature value $(7000 \mathrm{~K})$. However, for that common temperature value, a good agreement is found when comparing the resulting non-LTE corrections for the high-excitation C I lines; at $\log g=3.5, \Delta \log \epsilon_{\mathrm{C}}$ reaches $\sim-0.45 \mathrm{dex}$ in both studies. For a given stellar model, we too find increasing non-LTE corrections with line strength, see the trend indicated by identical symbols in Fig. 7: this effect has already been explained by Stürenburg \& Holweger (1990) as due to the numerical enhancement, in the flat part of the curve-of-growth, of the abundance correction derived from equivalent width changes due to non-LTE, compared to the abundance correction derived in the linear part of the curve-of-growth. When decreasing gravity or increasing effective temperature (see our Fig. 6, note that for $T_{\text {eff }} \gtrsim 5500 \mathrm{~K}$, the non-LTE abundance corrections tend to become more insensitive to temperature changes when fixing metallicity and gravity, except at very low metallicity), generally larger non-LTE corrections result for both studies. Another similarity between these two works - and also with that of Stürenburg \& Holweger (1990) - is that the four lowest levels of C I (not involved in the high-excitation transitions of interest here) in our model are found for almost all cases in the parameter space we study to have Boltzmann populations throughout the model atmosphere, due to strong collisional coupling between the neutral ground state and the next three lowest-lying levels. This ensures that the forbidden [C I] line at $872.7 \mathrm{~nm}$ (transition between the two lowest-lying levels in the singlet system) is in general not affected by departures from LTE. The levels involved in this transition only start to experience non-LTE effects at $[\mathrm{Fe} / \mathrm{H}]=-3$, for $T_{\text {eff }} \gtrsim 6500 \mathrm{~K}$ and higher (and increasing with decreasing gravity too). The two levels tend to experience rather similar departures from LTE. Rentzsch-Holm (1996b) similarly found that increas$\operatorname{ing} T_{\text {eff }}$ for the higher temperature and metallicities range in that study resulted in underpopulation of these levels: they showed this can transmit to other levels through line transitions and lead to global underpopulation of C I around $\sim 12000 \mathrm{~K}$. In our case, both the high temperature and low metallicity act to increase the magnitude and the atmospheric depth of the onset of departures from LTE, because the UV flux can more effectively underpopulate the atomic levels involved in the transition.

\section{Galactic chemical evolution of carbon}

Equipped with our non-LTE calculations, we have revisited the observational study of $\mathrm{C}$ and $\mathrm{O}$ in halo stars by Akerman et al. (2004). Those authors found that [C/O] seems to rise for $[\mathrm{O} / \mathrm{H}]<-1$; which could suggest high carbon yields from zerometallicity, so called Population III stars. They however assumed that the non-LTE abundance corrections for the two elements would be the same. We have calculated specific non-LTE corrections for their sample of 34 dwarf halo stars. Since in their analysis those authors used the same atmospheric models as we employ here, our derived non-LTE corrections can be directly applied to their LTE abundance results. Considering their estimate for random errors in equivalent widths measurements and stellar parameters determination - which in turn affects their resulting LTE abundances - and the uncertainty in our non-LTE calculations $(\sim 0.1 \mathrm{dex})$, we consider it feasible to study the $\mathrm{C}$ and $\mathrm{O}$ trends. Moreover, the $[\mathrm{C} / \mathrm{O}]$ ratio is essentially insensitive to changes in the stellar parameters, given that the $\mathrm{C}$ and $\mathrm{O}$ high-excitation abundance indicators used are affected in a similar way. We are thus confident that our results are a step forward with respect to previous works.

Table 2 lists the results obtained for the $\mathrm{C}$ and $\mathrm{O}$ abundances. Figure 9 shows the trend with atmospheric parameters of the carbon non-LTE corrections we obtained for the sample in Akerman et al. (2004). A clear increase in the non-LTE abundance corrections (becoming approximately 0.3 dex more negative at $[\mathrm{Fe} / \mathrm{H}] \sim-3$ than at $[\mathrm{Fe} / \mathrm{H}] \sim-1)$ is seen as one moves to low metallicity, which is mostly due to the higher effective temperatures of the sample stars towards the metal-poor regime ( $T_{\text {eff }}$ values increase by approximately $500 \mathrm{~K}$ or more for stars below $[\mathrm{Fe} / \mathrm{H}] \sim-2$, compared to the higher-metallicity stars in the sample). This is still in agreement with the fact that in general, when only decreasing the metallicity of the atmospheric model to $[\mathrm{Fe} / \mathrm{H}] \sim-3$, the non-LTE abundance corrections are 
Table 2. The IDs, atmospheric parameters (effective temperature, gravity and iron content) and the LTE abundances for oxygen and carbon for the sample of low-metallicity stars from Akerman et al. (2004) are shown in Cols. 1, 2, 3, 4, 5 and 7 respectively. The corresponding LTE [C/O] values are in Col. 10. Our adopted non-LTE abundance corrections for oxygen and carbon (obtained when neglecting collisions with $\mathrm{HI}$ atoms) and the resulting $[\mathrm{O} / \mathrm{H}]$ and $[\mathrm{C} / \mathrm{O}]$ non-LTE abundance ratios from this study are shown in Cols. 6, 8, 9 and 11 respectively. All values from the Akerman et al. study have been corrected, where appropriate, for our different choice of the carbon and oxygen solar abundances (log $\epsilon_{\mathrm{C}_{\odot}}=8.39$ and $\log \epsilon_{\mathrm{O}_{\odot}}=8.66$ ).

\begin{tabular}{|c|c|c|c|c|c|c|c|c|c|c|}
\hline Star & $\begin{array}{l}T_{\text {eff }} \\
{[\mathrm{K}]}\end{array}$ & $\begin{array}{l}\log g \\
{[\operatorname{cgs}]}\end{array}$ & $\begin{array}{c}{[\mathrm{Fe} / \mathrm{H}]} \\
(\mathrm{LTE})\end{array}$ & $\begin{array}{l}\log \epsilon_{\mathrm{O}} \\
\text { (LTE) }\end{array}$ & $\Delta \log \epsilon_{\mathrm{O}}$ & $\begin{array}{l}\log \epsilon_{\mathrm{C}} \\
(\mathrm{LTE})\end{array}$ & $\Delta \log \epsilon_{\mathrm{C}}$ & $\begin{array}{c}{[\mathrm{O} / \mathrm{H}]} \\
\text { (non-LTE) }\end{array}$ & $\begin{array}{l}{[\mathrm{C} / \mathrm{O}]} \\
\text { (LTE) }\end{array}$ & $\begin{array}{c}{[\mathrm{C} / \mathrm{O}]} \\
\text { (non-LTE) }\end{array}$ \\
\hline$\overline{B D}-13^{\circ} 3442$ & 6500 & 4.16 & -2.61 & 6.85 & $-0.18^{*}$ & 6.13 & -0.37 & $-1.99 *$ & -0.45 & $-0.64 *$ \\
\hline CD-30 18140 & 6272 & 4.13 & -1.88 & 7.55 & -0.13 & 6.72 & -0.26 & -1.24 & -0.56 & -0.69 \\
\hline CD-35ำ 14849 & 6125 & 4.11 & -2.41 & 7.12 & -0.11 & 6.40 & -0.30 & -1.65 & -0.45 & -0.64 \\
\hline $\mathrm{CD}-42^{\circ} 14278$ & 5812 & 4.25 & -2.12 & 7.42 & -0.13 & 6.62 & -0.22 & -1.37 & -0.53 & -0.62 \\
\hline HD 103723 & 6040 & 4.26 & -0.82 & 8.34 & -0.20 & 7.63 & -0.23 & -0.52 & -0.44 & -0.47 \\
\hline HD 105004 & 5919 & 4.36 & -0.86 & 8.26 & -0.19 & 7.75 & -0.22 & -0.59 & -0.24 & -0.27 \\
\hline HD 106038 & 5919 & 4.30 & -1.42 & 8.08 & -0.18 & 7.40 & -0.27 & -0.76 & -0.41 & -0.50 \\
\hline HD 108177 & 6034 & 4.25 & -1.74 & 7.80 & -0.15 & 6.94 & -0.24 & -1.01 & -0.59 & -0.68 \\
\hline HD 110621 & 5989 & 3.99 & -1.66 & 7.95 & -0.17 & 7.06 & -0.28 & -0.88 & -0.62 & -0.73 \\
\hline HD 121004 & 5595 & 4.31 & -0.77 & 8.71 & -0.23 & 8.05 & -0.20 & -0.18 & -0.39 & -0.36 \\
\hline HD 140283 & 5690 & 3.69 & -2.42 & 7.11 & -0.11 & 6.34 & -0.26 & -1.66 & -0.50 & -0.65 \\
\hline HD 146296 & 5671 & 4.17 & -0.74 & 8.53 & -0.22 & 7.96 & -0.24 & -0.35 & -0.30 & -0.32 \\
\hline HD 148816 & 5823 & 4.14 & -0.73 & 8.65 & -0.22 & 8.02 & -0.25 & -0.23 & -0.36 & -0.39 \\
\hline HD 160617 & 5931 & 3.77 & -1.79 & 7.42 & -0.13 & 6.74 & -0.28 & -1.37 & -0.41 & -0.56 \\
\hline HD 179626 & 5699 & 3.92 & -1.14 & 8.46 & -0.22 & 7.62 & -0.25 & -0.42 & -0.57 & -0.60 \\
\hline HD 181743 & 5863 & 4.32 & -1.93 & 7.66 & -0.14 & 6.82 & -0.21 & -1.14 & -0.57 & -0.64 \\
\hline HD 188031 & 6054 & 4.03 & -1.79 & 7.75 & -0.15 & 6.87 & -0.26 & -1.06 & -0.61 & -0.72 \\
\hline HD 193901 & 5672 & 4.38 & -1.12 & 8.16 & -0.18 & 7.41 & -0.20 & -0.68 & -0.48 & -0.50 \\
\hline HD 194598 & 5906 & 4.25 & -1.17 & 8.19 & -0.19 & 7.48 & -0.23 & -0.66 & -0.44 & -0.48 \\
\hline HD 215801 & 6005 & 3.81 & -2.29 & 7.22 & -0.12 & 6.34 & -0.30 & -1.56 & -0.61 & -0.79 \\
\hline LP815-43 & 6533 & 4.25 & -2.67 & 6.61 & $-0.25^{*}$ & 6.14 & -0.39 & $-2.30 *$ & -0.20 & $-0.34 *$ \\
\hline G011-044 & 5995 & 4.29 & -2.09 & 7.47 & -0.13 & 6.63 & -0.22 & -1.32 & -0.57 & -0.66 \\
\hline G013-009 & 6360 & 4.01 & -2.27 & 7.15 & -0.11 & 6.47 & -0.35 & -1.62 & -0.41 & -0.65 \\
\hline G016-013 & 5602 & 4.17 & -0.76 & $8.75^{\dagger}$ & -0.20 & $8.00^{\dagger}$ & -0.22 & $-0.11^{\dagger}$ & -0.48 & $-0.50^{\dagger}$ \\
\hline G018-039 & 5910 & 4.09 & -1.52 & 8.12 & -0.18 & 7.29 & -0.25 & -0.72 & -0.56 & -0.63 \\
\hline G020-008 & 5855 & 4.16 & -2.28 & 7.53 & -0.13 & 6.66 & -0.24 & -1.26 & -0.60 & -0.71 \\
\hline G024-003 & 5910 & 4.16 & -1.67 & 7.62 & -0.14 & 6.71 & -0.22 & -1.18 & -0.64 & -0.72 \\
\hline G029-023 & 5966 & 3.82 & -1.80 & 7.79 & -0.15 & 6.85 & -0.28 & -1.02 & -0.67 & -0.80 \\
\hline G053-041 & 5829 & 4.15 & -1.34 & 7.86 & -0.16 & 7.07 & -0.25 & -0.96 & -0.52 & -0.61 \\
\hline G064-012 & 6511 & 4.39 & -3.17 & 6.44 & $-0.30 *$ & 5.68 & -0.45 & $-2.52 *$ & -0.49 & $-0.64 *$ \\
\hline G064-037 & 6318 & 4.16 & -3.12 & 6.44 & $-0.30 *$ & 5.72 & -0.42 & $-2.52 *$ & -0.45 & $-0.57 *$ \\
\hline G066-030 & 6346 & 4.24 & -1.52 & 7.93 & -0.13 & 6.93 & -0.27 & -0.89 & -0.73 & -0.87 \\
\hline G126-062 & 5943 & 3.97 & -1.64 & 7.98 & -0.17 & 7.02 & -0.27 & -0.85 & -0.69 & -0.79 \\
\hline G186-026 & 6273 & 4.25 & -2.62 & 6.71 & $-0.15^{*}$ & 6.14 & -0.33 & $-2.10 *$ & -0.30 & $-0.48 *$ \\
\hline
\end{tabular}

* Note that the O I non-LTE abundance corrections for the five most metal-poor stars have been revised with respect to those given in Akerman et al. (2004), as explained in the text. ${ }^{\dagger}$ The $\mathrm{C}$ and $\mathrm{O}$ abundances of G016-013 have been revised by +0.4 dex with respect to those in Akerman et al. (2004), due to the fact that the wrong (hotter) stellar model for HD 160617 was accidentally used in that analysis instead of the correct one for this star (P. E. Nissen, private communication). This affects $[\mathrm{O} / \mathrm{H}]($ and $[\mathrm{C} / \mathrm{H}])$ but leaves $[\mathrm{C} / \mathrm{O}]$ intact for this star. We trust that the stellar parameters for this star in Akerman et al. (also given here) have been correctly derived in their analysis.

roughly of the same order than at solar metallicity (see Figs. 6 and 7). A similar increase below $[\mathrm{Fe} / \mathrm{H}] \sim-1$ in the non-LTE abundance corrections of Akerman et al.'s sample of halo stars was found by Takeda \& Honda (2005), however when we adopt their same choice for the $\mathrm{H}$ collision efficiency $\left(S_{\mathrm{H}}=1\right)$ and microturbulence $\left(\xi=1 \mathrm{~km} \mathrm{~s}^{-1}\right)$, we still find corrections that are more negative than theirs by $\sim 0.05-0.10$ dex for the lowestmetallicity stars in that sample.

Previous studies of Milky Way disk stars (e.g. Gustafsson et al. 1999) found a slowly decreasing [C/Fe] with increasing metallicity (slope of -0.17 dex per dex). However, very recently, Bensby \& Feltzing (2006), analysing a sample of thin and thick disk stars and using [C I] and [O I] lines immune from non-LTE effects, found a more or less flat trend from around solar metallicity down to $[\mathrm{Fe} / \mathrm{H}] \simeq-0.9$. We find a roughly solar $[\mathrm{C} / \mathrm{Fe}]$ (or slightly higher when adopting $S_{\mathrm{H}}=1$ ) extending much further down to $[\mathrm{Fe} / \mathrm{H}] \simeq-3.2$ (Fig. 10), albeit with a rather large and - at least in part - likely real scatter. The plot shows how the $\mathrm{LTE}[\mathrm{C} / \mathrm{Fe}]$ values in the Akerman et al. study have a large scatter around a value of $\sim+0.35 \mathrm{dex}$, with a possible hint for a slight increase with decreasing $[\mathrm{Fe} / \mathrm{H}]$. This trend is removed when correcting for our increasingly large (as one moves down in metallicity) non-LTE corrections for the stars in their sample. In Fig. 10 we also show $[\mathrm{O} / \mathrm{Fe}]$ as a function of $[\mathrm{Fe} / \mathrm{H}]$ for the halo stars of Akerman et al. As for the $[\mathrm{C} / \mathrm{Fe}]$ versus $[\mathrm{Fe} / \mathrm{H}]$ plot, the disk stars of Bensby \& Feltzing are included too. The 


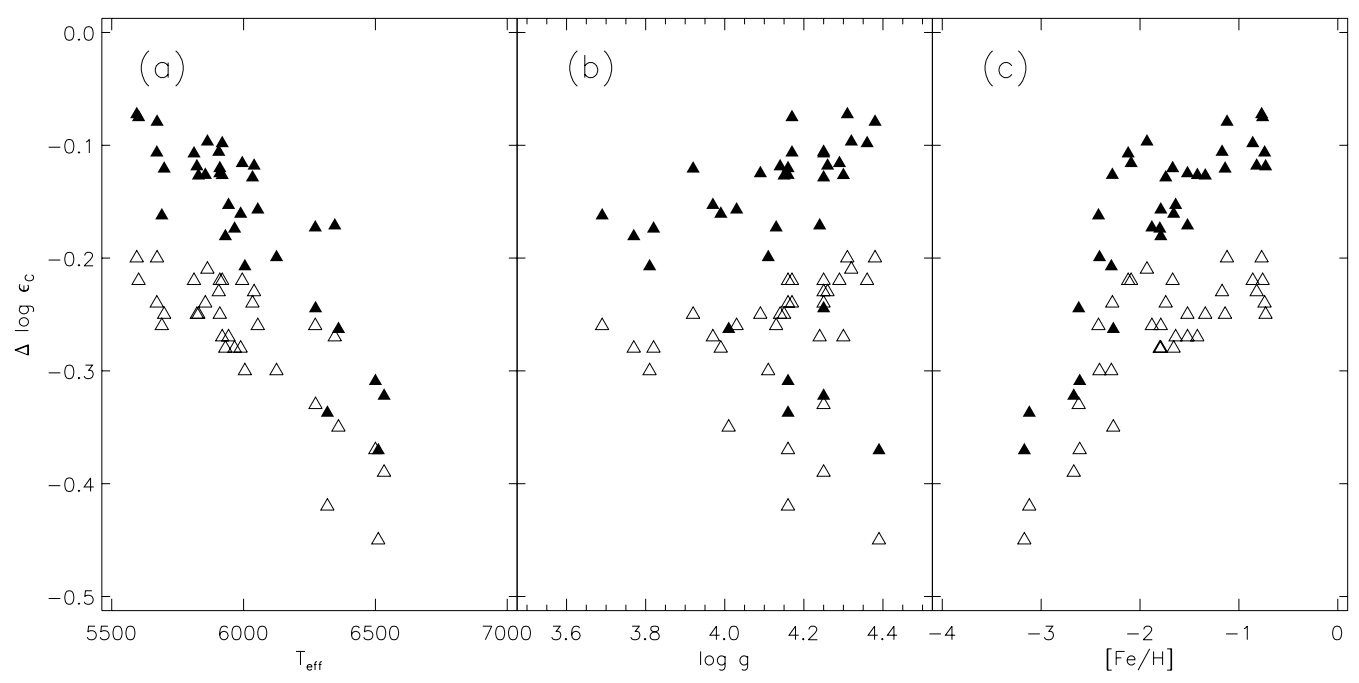

Fig. 9. The non-LTE abundance corrections (empty triangles: $S_{\mathrm{H}}=0$; filled triangles: $S_{\mathrm{H}}=1$ ) for the 34 halo stars in the sample by Akerman et al. (2004) as a function of $\left.T_{\text {eff }} \mathbf{a}\right), \log g$ b) and $\left.[\mathrm{Fe} / \mathrm{H}] \mathbf{c}\right)$.
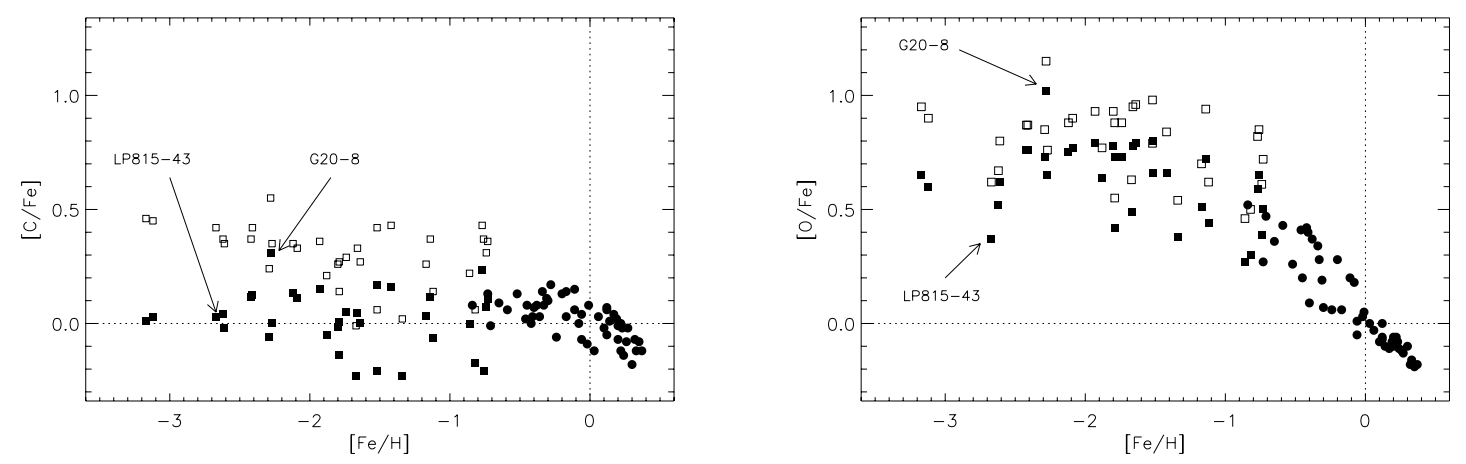

Fig. 10. Trends of $[\mathrm{C} / \mathrm{Fe}]$ vs. $[\mathrm{Fe} / \mathrm{H}]$ (left panel) and $[\mathrm{O} / \mathrm{Fe}]$ vs. $[\mathrm{Fe} / \mathrm{H}]$ (right panel) in Milky Way halo (squares) and disk stars (circles, Bensby \& Feltzing 2006, from [CI] and [O I] lines free from non-LTE effects). The LTE values from Akerman et al. (2004), corrected for our different choice of carbon and oxygen solar abundances, are represented as empty squares in the two panels, while the non-LTE abundances are shown as filled squares. The non-LTE results are those obtained when neglecting collisions with hydrogen. Note that the $[\mathrm{O} / \mathrm{Fe}]$ non-LTE values in the right plot for the five most metal-poor stars already include the new OI non-LTE abundance corrections we present here (see text).

original results of Akerman et al. have here been corrected for our revised O I non-LTE corrections at the lowest $[\mathrm{Fe} / \mathrm{H}]$ as well as for our different solar abundances (Asplund et al. 2005b). The $[\mathrm{O} / \mathrm{Fe}]$ trend in metal-poor stars is still a hotly debated topic and a full account of this problem is beyond the scope of the present paper; we refer the reader to recent discussions such as Nissen et al. (2002) and Asplund (2005) and references therein. We note however that Fig. 10 suggests a roughly flat trend at a level $[\mathrm{O} / \mathrm{Fe}] \sim+0.6$ with no indication of a linear trend with slope of $\sim-0.3 \mathrm{dex} / \mathrm{dex}$ as claimed by Israelian et al. (1998) and Boesgaard et al. (1999). Asplund \& García Pérez (2001) have argued based on 3D hydrodynamical model atmospheres of metalpoor stars that $\mathrm{O}$ abundances derived from $\mathrm{OH}$ lines as used by Israelian et al. and Boesgaard et al. can be severely overestimated in 1D (see also Collet et al. 2006). noted:

A few peculiar cases in the two plots in Fig. 10 should be

- the C and O LTE abundances for G16-13 $([\mathrm{Fe} / \mathrm{H}]=-0.76)$ are both increased by 0.4 dex with respect to Akerman et al. (2004), due to the fact that a too hot stellar model was used in their analysis for this star (P. E. Nissen, private communication). This moves the star from the lower to the upper extreme of the distribution in the $[\mathrm{C} / \mathrm{Fe}]$ and $[\mathrm{O} / \mathrm{Fe}]$ versus $[\mathrm{Fe} / \mathrm{H}]$ plots. Because the scatter in both plots is rather large, this does not significantly affect the overall resulting trends. Moreover, since both the $\mathrm{C}$ and $\mathrm{O}$ abundances are increased by the same amount, the star maintains the same $[\mathrm{C} / \mathrm{O}]$ as in Akerman et al. (demonstrating how insensitive [C/O] is to the model used), while $[\mathrm{O} / \mathrm{H}]$ increases by 0.4 dex: the overall $[\mathrm{C} / \mathrm{O}]$ trend is unchanged too, again because of masking by the relatively large scatter at the higher-metallicity extreme of the distribution, to which the star is shifted.

- the two most extreme cases in Fig. 10 are LP815-43 (with a low $[\mathrm{O} / \mathrm{Fe}]_{\text {non-LTE }}=0.37$ ) and $\mathrm{G} 20-8$ (showing very high $[\mathrm{C} / \mathrm{Fe}]_{\text {non-LTE }}=0.31$ and $\left.[\mathrm{O} / \mathrm{Fe}]_{\text {non-LTE }}=1.02\right)$. We note that for the O I triplet equivalent widths in LP815-43, Nissen et al. (2002) give different values than those in Akerman et al. (2004). The smaller line-to-line scatter in the former determination seems to better correspond to the variation in gf-values; moreover, the $\mathrm{S} / \mathrm{N}$ for this star is higher in Nissen et al.'s spectrum. These authors derive $\log \epsilon_{\mathrm{O}}=6.71$ compared to $\log \epsilon_{\mathrm{O}}=6.61$ in Akerman et al. (2004). It is thus possible that the oxygen abundance derived in the latter work needs to be increased by up to $0.1 \mathrm{dex}$, with the 
errors in the equivalent width determinations likely attributable to e.g. continuum placement problems. The case of $\mathrm{G} 20-8$, which shows anomalously large $[\mathrm{C} / \mathrm{Fe}]$ and $[\mathrm{O} / \mathrm{Fe}]$ values (by $\sim 0.3 \mathrm{dex}$ ), seems to indicate that its temperature might be underestimated by as much as $400 \mathrm{~K}$, possibly due to uncertainties in the reddening.

The O I non-LTE abundance corrections are taken from Akerman et al. (2004), with the exception of the five most metal-poor stars. Akerman et al. interpolated the non-LTE corrections of Nissen et al. (2002) as a function of O I equivalent width, leading them to deduce a non-LTE correction of -0.10 dex for those five stars. We have carried out non-LTE calculations in the same manner as described by Nissen et al. Our corrections for the five most metal-poor stars are more negative than found by Akerman et al. (2004), varying between -0.18 and -0.30 dex. Moreover, while - with respect to [C/O] - we find that the non-LTE corrections for carbon and oxygen almost balance each other at higher metallicity, at $[\mathrm{O} / \mathrm{H}] \lesssim-1$ they become more negative for carbon. While the $\mathrm{O}$ I non-LTE corrections are typically $\simeq-0.2 \mathrm{dex}$, and -0.3 dex at most at $[\mathrm{Fe} / \mathrm{H}] \simeq-3$, the corresponding $\mathrm{C}$ I nonLTE corrections are $\simeq 0.15$ dex lower still at these low $[\mathrm{Fe} / \mathrm{H}]$. We adopt slightly different solar $\mathrm{C}$ and $\mathrm{O}$ abundances than done by Akerman et al. ${ }^{8}$, which also slightly modifies the resulting $[\mathrm{C} / \mathrm{H}],[\mathrm{O} / \mathrm{H}]$ and $[\mathrm{C} / \mathrm{O}]$ values. Had we used the solar values determined with a 1D MARCS model atmosphere instead, [O/H] would have been lower by $-0.05 \mathrm{dex}$ and $[\mathrm{C} / \mathrm{H}]$ higher by +0.04 dex only, for all stars. Clearly, such a small and constant offset is not in any way crucial in determining the general trends of $[\mathrm{C} / \mathrm{Fe}]$ and $[\mathrm{O} / \mathrm{Fe}]$, because all stars would shift by the same amount. More importantly, the $[\mathrm{C} / \mathrm{O}]$ values remain essentialy unchanged. Indeed, we recover a similar trend to that in the work by Akerman et al. for the $[\mathrm{C} / \mathrm{O}]$ abundance ratio at low metallicity, even though at a lower level (see Fig. 11). We note that the similarity is due to the fact that the carbon non-LTE corrections in our study are more negative at low metallicity than those we estimate for oxygen, by approximately the same amount for all of the metal-poor stars below $[\mathrm{O} / \mathrm{H}] \sim-1.5$. This gives a more or less constant downward shift of those $[\mathrm{C} / \mathrm{O}]$ values as compared to the Akerman et al. study, who had assumed for carbon that the same non-LTE corrections would applicable as for oxygen. The revised (more negative) O I non-LTE abundance corrections we use for the five most metal-poor stars in the sample partially compensate the large non-LTE corrections we find for C I at such low metallicities. These increased non-LTE corrections for oxygen also shift those stars to correspondingly lower $[\mathrm{O} / \mathrm{H}]_{\text {non-LTE }}$ values with respect to the original study by Akerman et al., "stretching" the low-metallicity part of the plot with respect to their work.

In summary, the adoption of the recent values of solar abundance and the application of our carbon and oxygen non-LTE corrections has the net effect of shifting down the $[\mathrm{C} / \mathrm{O}]$ values of the low-metallicity stars by similar amounts, preserving the possible $[\mathrm{C} / \mathrm{O}]$ upturn at $[\mathrm{O} / \mathrm{H}] \lesssim-1$, although at a lower $[\mathrm{C} / \mathrm{O}]$ level. We do urge that this rising trend should not be overinterpreted at this stage. First of all, if our suspicion is confirmed that the oxygen abundance has been underestimated by $\sim 0.1 \mathrm{dex}$ by Akerman et al. for LP815-43 - which is the star with the highest $[\mathrm{C} / \mathrm{O}]$ ratio at low metallicity - it would imply that this star

\footnotetext{
8 Namely, $\log \epsilon_{C_{\odot}}=8.39$ and $\log \epsilon_{O_{\odot}}=8.66$ (Asplund et al. 2004; Asplund et al. 2005b), as compared to $\log \epsilon_{C_{\odot}}=8.41$ and $\log \epsilon_{O_{\odot}}=$ 8.74 in Akerman et al. (2004). We believe the values we adopt are best estimates since they are averaged over different lines and they account for 3D/non-LTE effects.
}

will accordingly have a lower $[\mathrm{C} / \mathrm{O}]$, bringing it closer to the rest of the low-metallicity points and giving in the end a less significant indication for the claimed upturn. The $[\mathrm{C} / \mathrm{O}]$ trend below $[\mathrm{O} / \mathrm{H}] \sim-1$ might thus have a gradual increase with a shallow slope of $\sim-0.2$ dex/dex. Moreover, in view of the large departures from LTE we found for C I in 1D, it is important to carry out 3D non-LTE calculations. Furthermore, our tests have revealed that the $\mathrm{O}$ I non-LTE effects at these very low $[\mathrm{Fe} / \mathrm{H}]$ may be more uncertain than previously thought due to large sensitivity to poorly known collisional cross-sections. We are indeed planning to investigate this in an upcoming work. Finally, the $T_{\text {eff }}$ scale for halo stars is still under debate (e.g. Meléndez et al. 2006). As mentionned, the $[\mathrm{C} / \mathrm{O}]$ ratio should be relatively unaffected, given the similar temperature dependence of the $\mathrm{C}$ and $\mathrm{O}$ lines we employ. However, the results could be influenced indirectly through the different temperature dependence of the nonLTE corrections for the two elements. Given all of the above, the claim of carbon production from high-mass Pop. III stars is thus still uncertain in our opinion.

Spite et al. (2005) used different abundance indicators (namely, the $\mathrm{G}$ band of $\mathrm{CH}$ and the forbidden [O I] line at 630.0 $\mathrm{nm})$ to extend the study by Akerman et al. down to $[\mathrm{Fe} / \mathrm{H}] \sim-4$ $([\mathrm{O} / \mathrm{H}] \sim-3)$, again deriving an increasing $[\mathrm{C} / \mathrm{O}]$ ratio reaching a near solar level ( -0.2 dex) toward lower metallicity. However, they did not account for 3D effects which should affect the carbon molecular lines they employed more than the [O I] line they used. Even though those authors studied extremely metal-poor halo giants, for which 3D effects are not well known, one can use the results for turn-off stars at similarly low metallicity as an indication, so that the corresponding values given in Spite et al. might turn out to be overestimated by $\sim 0.3$ dex or more (Asplund 2005) and the [C/O] trend could still be roughly constant or only slowly increasing.

\section{Conclusions}

We have carried out non-LTE spectral-line formation calculations for C I absorption features. The computations have been performed on a grid of 168 1D MARCS stellar model atmospheres with varying parameters $\left(4500 \leq T_{\mathrm{eff}} \leq 7000 \mathrm{~K}\right.$, $2 \leq \log g \leq 5,-3 \leq[\mathrm{Fe} / \mathrm{H}] \leq 0)$ and $-0.60 \leq[\mathrm{C} / \mathrm{Fe}] \leq+0.60)$. The main results are:

- We generally find large, negative non-LTE abundance corrections for high-excitation neutral carbon permitted lines, i.e. LTE abundances are too large. At solar metallicity, the dominant non-LTE effect is that of the line source function dropping below the Planckian mostly due to under-populated upper atomic levels in the transitions, but at low metallicity it is that of line opacity due to overpopulated lower levels.

- We find substantial non-LTE abundance corrections for metal-poor halo turn-off stars: typically $\sim-0.4 \mathrm{dex}$ at $[\mathrm{Fe} / \mathrm{H}] \sim-3$; these results are relatively immune to the adopted $\mathrm{H}$ collisional efficiency.

- Using our resulting non-LTE corrections, we revisit the observational study of Akerman et al. (2004) and find that the $[\mathrm{C} / \mathrm{O}]$ trend at low metallicity might still be suggesting an upturn indicating possible production of carbon through nucleosynthesis in Pop. III stars. We however warn that more observational data is needed - together with detailed nonLTE calculations for oxygen, to understand the magnitude of the abundance corrections applicable for this element at metallicities $[\mathrm{Fe} / \mathrm{H}] \lesssim-2.5-$ in order to reduce the still large uncertainties and finally settle this issue. 

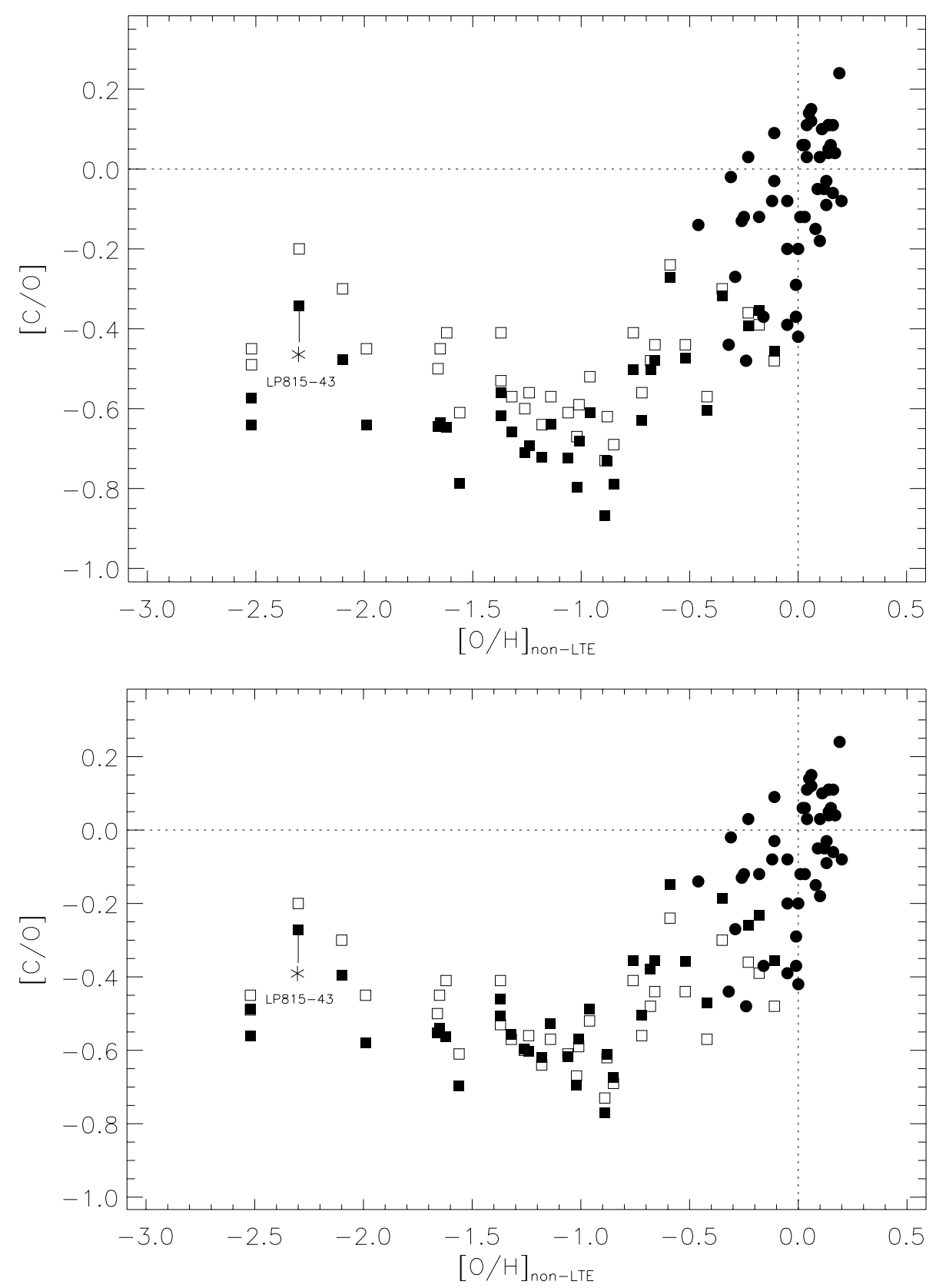

Fig. 11. Trend of $[\mathrm{C} / \mathrm{O}]$ vs. $[\mathrm{O} / \mathrm{H}]$ in Milky Way halo and disk stars. The upper panel represents the new observational trends obtained when our non-LTE calculations are applied to the data in Akerman et al. (2004) (using our different choice of carbon and oxygen solar abundances and assuming negligible collisions with hydrogen). The $[\mathrm{O} / \mathrm{H}]$ values already take into account our more negative non-LTE corrections for oxygen at low metallicity. The empty and filled squares represent the [C/O] LTE and non-LTE values respectively. The lower panel uses the same notation, with results now calculated accounting for hydrogen collisions for carbon (with a choice of $S_{\mathrm{H}}=1$ ). In both panels, the starred symbol indicates the $[\mathrm{C} / \mathrm{O}]$ value we believe is more realistic for LP815-43 (see discussion in text).

Without doubt one of the necessary steps for obtaining reliable stellar abundances in future large scale surveys will be to perform a vast quantity of detailed non-LTE studies for an increased number of different chemical elements and stellar parameters. Very many precise atomic input data for collisional and radiative processes have to be known for realistic results. The use of sophisticated techniques for quantum-mechanical computations have led to accurate atomic radiative data fortunately becoming available in recent years to the astrophysical community, thanks to such large scale efforts as e.g. the Opacity and IRON Projects. We underline the necessity for more theoretical and laboratory studies aimed at obtaining such accurate atomic data and in particular at improving our knowledge on the correct treatment of the still poorly understood collisions with electrons and with hydrogen atoms. The effect of using 3D hydrodynamical model atmospheres should be investigated as the 3D nonLTE effects could prove to be even stronger than in 1D, due to the presence of large temperature inhomogeneities and to the different temperature structure in the optically thin atmospheric layers with respect to 1D hydrostatic models, especially for the lowmetallicity regime (Asplund et al. 1999).

Acknowledgements. D.F. acknowledges support from RSAA, in the form of a Joan Duffield Research Scholarship and an Alex Rodgers Travelling Scholarship and is grateful to the Institute of Theoretical Astrophysics, University of Oslo, Norway, for its repeated hospitality. D.F. also thanks Øystein Langangen and Jorge Meléndez for useful discussions and Poul Erik Nissen for careful 
reading of this manuscript. This work has been partly funded by the Australian Research Council (grants DP0342613 and DP0558836) and also supported by the Research Council of Norway through grant 146467/420 and through a grant of computing time from the Programme for Supercomputing. This research has made extensive use of NASA's Astrophysics Data System and of the NIST Atomic Spectra Database, which is operated by the National Institute of Standards and Technology. Finally, we would like to thank the referee for remarks that were useful in improving the paper.

\section{References}

Akerman, C. J., Carigi, L., Nissen, P. E., et al. 2004, A\&A, 414, 931

Allende Prieto, C., Asplund, M., López, Ramón, J. G., \& Lambert, D. L. 2002, ApJ, 567, 544

Andersson, H., \& Edvarsson, B. 1994, A\&A, 290, 590

Arnett, D. 1996, Supernovae and Nucleosynthesis (NJ: Princeton University, Princeton)

Asplund, M. 2005, ARA\&A, 43, 481

Asplund, M., \& García Pérez, A. E. 2001, A\&A, 372, 601

Asplund, M., Gustafsson, B., Kiselman, D., \& Eriksson, K. 1997, A\&A, 318, 521

Asplund, M., Nordlund, Å., Trampedach, R., \& Stein, R. F. 1999, A\&A, 346, L17

Asplund, M., Grevesse, N., Sauval, A. J., et al. 2004, A\&A, 417, 751

Asplund, M., Grevesse, N., Sauval, A. J., et al. 2005a, A\&A, 431, 693

Asplund, M., Grevesse, N., \& Sauval, A. J. 2005b, ASP Conf. Ser., 336, 25

Ballero, S. K, Matteucci, F., \& Chiappini, C. 2006, New Astron., 11, 306

Becker, G. D., Sargent, W. L. W., Rauch, M., \& Simcoe, R. A. 2006, ApJ, 640, 69

Belyaev, A. K., \& Barklem, P. S. 2003, Phys. Rev. A, 68, 62703

Belyaev, A. K., Grosser, J., Hahne, J., \& Menzel, T. 1999, Phys. Rev. A, 60, 2151

Bensby, T., \& Feltzing, S. 2006, MNRAS, 367, 1181

Boesgaard, A. M., King, J. R., Deliyannis, C. P., \& Vogt, S. S. 1999, AJ, 117, 492

Burbidge, E. M., Burbidge, G. R., Fowler, W. A., \& Hoyle, F. 1957, Rev. Mod. Phys., 29, 547

Carigi, L., Peimbert, M., Esteban, C., \& García-Rojas, J. 2005, ApJ, 623, 213

Carlsson, M. 1986, Uppsala Astron. Obs. Rep. No. 33

Carlsson, M. 1992, ASP Conf. Ser., 26, 499

Carlsson, M., Rutten, R. J., Bruls, J. H. M. J., \& Shchukina, N. G. 1994, A\&A, 288,860

Chiappini, C., Matteucci, F., \& Gratton, R. 1997, ApJ, 477, 765

Chiappini, C., Romano, D., \& Matteucci, F. 2003, MNRAS, 339, 63

Chiappini, C., Hirschi, R., Meynet, G., et al. 2006, A\&A, 449, L27

Collet, R., Asplund, M., \& Trampedach, R. 2006, ApJ, 644, L121

Cunto, W., Mendoza, C., Ochsenbein, F., \& Zeippen, C. 1993, A\&A, 275, L5
Dopita, M. A., \& Sutherland, R. S. 2003, Astrophysics of the diffuse universe (Springer), 2003

Drawin, H. W. 1968, Z. Phys., 211, 404

Erni, P., Richter, P., Ledoux, C., \& Petitjean, P. 2006, A\&A, 451, 19

Fabbian, D., Asplund, M., Carlsson, M., \& Kiselman, D. 2005, in From Lithium to Uranium: Elemental Tracers of Early Cosmic Evolution, ed. Hill François, \& Primas (Cambridge University Press), IAU Symp., 228, 255

Fleck, I., Grosser, J., Schnecke, A., et al. 1991, J. Phys. B, 24, 4017

Gavilán, M., Buell, J. F., \& Mollá, M. 2005, A\&A, 432, 861

Gray, D. F. 1992, The Observation and Analysis of Stellar Photospheres, 2nd edn. (Cambridge University Press)

Gustafsson, B., Bell, R. A., Eriksson, K., \& Nordlund, Å. 1975, A\&A, 42, 407

Gustafsson, B., Karlsson, T., Olsson, E., et al. 1999, A\&A, 342, 426

Hibbert, A., Biemont, E., Godefroid, M., \& Vaeck, N. 1993, A\&AS, 99, 179

Israelian, G., García López, R. J., \& Rebolo, R. 1998, ApJ, 507, 805

Kaulakys, B. 1985, J. Phys. B, 18, L167

Levshakov, S. A., Centurión, M., Molaro, P., \& Kostina, M. V. 2006, A\&A, 447, L21

Luo, D., \& Pradhan, A. K. 1989, J. Phys. B, 22, 3377

Magain, P. 1986, A\&A, 163, 135

Meléndez, J., Shchukina, N. G., Vasiljeva, I., \& Ramírez, I. 2006, ApJ, 642, 1082

Meynet, G., Ekström, S., \& Maeder, A. 2006, A\&A, 447, 623

Martin, W. C., Dalton, G. R., Fuhr, J. R., et al. 1995, ASP Conf. Ser., 81, 597

Mihalas, D., \& Mihalas, B. W. 2000, Foundations of Radiation Hydrodynamics (Dover Publications)

Nissen, P. E., Primas, F., Asplund, M., \& Lambert, D. L. 2002, A\&A, 390, 235

Pagel, B. E. J. 1997, Nucleosynthesis and Chemical Evolution of Galaxies (Cambridge, England: Cambridge University Press)

Paunzen, E., Kamp, I., Iliev, I. Kh., et al. 1999, A\&A, 345, 597

Reddy, B. E., Tomkin, J., Lambert, D. L., \& Allende Prieto, C. 2003, MNRAS, 340,304

Reddy, B. E., Lambert, D. L., \& Allende Prieto, C. 2006, MNRAS, 367, 1329

Rentzsch-Holm, I. 1996a, Model Atmospheres and Spectrum Synthesis, ed. Adelman, Kupka \& Weiss, ASP Conf. Ser., 108, 99

Rentzsch-Holm, I. 1996b, A\&A, 312, 966

Rutten, R. J. 2003, Radiative Transfer in Stellar Atmospheres http://www.phys.uu.nl/ rutten/Astronomy_lecture.html

Spite, M., Cayrel, R., Plez, B., et al. 2005, A\&A, 430, 655

Steenbock, W., \& Holweger, H. 1984, A\&A, 130, 319

Stürenburg, S., \& Holweger, H. 1990, A\&A, 237, 125

Takeda, Y. 1992, PASJ, 44, 649

Takeda, Y. 1994, PASJ, 46, 53

Takeda, Y., \& Honda, S. 2005, PASJ, 57, 65

Tomkin J., Lemke, M., Lambert, D. L., \& Sneden, C. 1992, AJ, 104, 1568

van Regemorter, H. 1962, ApJ, 136, 906

Wiese, W. L., Fuhr, J. R., \& Deters, T. M. 1996, J. Phys. Chem. Ref. Data Monograph, No. 7

Woosley, S. E., \& Weaver, T. A. 1995, ApJS, 101, 181 